\title{
Investigation of Deformation Dynamics in a Wrought Magnesium Alloy
}

\author{
Wei $\mathrm{Wu}^{1,2}$, Hua Qiao ${ }^{3}, \mathrm{Ke} \mathrm{An}^{2, *}$, Xiaoqian $\mathrm{Guo}^{3,4}$, Peidong $\mathrm{Wu}^{3}$, and Peter K. Liaw ${ }^{1, *}$
}

1. Department of Materials Science and Engineering, The University of Tennessee, Knoxville, TN 37996, USA

2. Chemical and Engineering Materials Division, Oak Ridge National Laboratory, Oak Ridge, TN 37831, USA

3. Department of Mechanical Engineering, McMaster University, Hamilton, ON, Canada L8S 4L7

4. State Key Laboratory for Geomechanics and Deep Underground Engineering, China University of Mining and Technology, Xuzhou, Jiangsu 221116, P. R. China

\section{*Corresponding authors}

Ke An, Ph.D.

Lead Scientist

Chemical and Engineering Materials Division

Oak Ridge National Laboratory

1 Bethel Valley Rd

Oak Ridge

TN 37831, USA

Office: +1-865-919-5226

Fax: +1-865-574-6080

E-mail: kean@ornl.gov

Peter K. Liaw, Ph.D.

Professor and Ivan Racheff Chair of Excellence

Dept. of Materials Science \& Engineering

The University of Tennessee

406 Ferris Hall

Knoxville

TN 37996, USA

Office: +1-865-974-6356

Fax: +1-865-974-4115

E-mail: pliaw@utk.edu 


\begin{abstract}
:
In the present research, the real-time in-situ neutron diffraction measurements under a continuous-loading condition and elastic-viscoplastic self-consistent (EVPSC) polycrystal modeling were employed to study the deformation dynamics and the effect of the deformation history on plastic deformation in a wrought magnesium alloy. The experimental results reveal that pre-deformation delays the activation of the tensile twinning during the subsequent compression, mainly resulting from the residual strains. Detwinning does not occur until the applied stress exceeds the tensile yield strength during reverse loading. It is believed that the grain rotation plays an important role in the elastic region during reverse loading. The EVPSC model, which has been recently updated by implementing the twinning and detwinning model, was employed to characterize the deformation mechanism during strain-path changes. The simulation result predicts well the experimental observation from the real-time in-situ neutron diffraction measurements. The present study provides a new insight of the nature of deformation mechanisms in a hexagonal close-packed (HCP) structured polycrystalline wrought magnesium alloy, which has significant implications for future work on studying the deformation mechanisms of HCP structured materials.
\end{abstract}

Keyword: magnesium alloy; neutron diffraction; continuous loading; EVPSC model; deformation mechanisms; twinning 


\section{Introduction}

Magnesium ( $\mathrm{Mg}$ ) alloys draw great attention to the science and engineering societies and industries, due to the combined properties of light weight, high strength-to-weight ratio, and high specific stiffness (Polmear, 1994; Aghion and Bronfin, 2000; Mordike and Ebert, 2001; Agnew, 2004; Agnew and Nie, 2010; Wu et al., 2012). In terms of structural components, wrought Mg alloys hold greater advantages over cast $\mathrm{Mg}$ alloys based on their superior mechanical properties (Doege and Droder, 2001; Park et al., 2003; Chen et al., 2007). Moreover, owing to their defectfree nature, the research on the mechanical behavior of wrought $\mathrm{Mg}$ alloys may shed light on the intrinsic plastic deformation mechanism of Mg alloys (Yang et al., 2008).

The wrought $\mathrm{Mg}$ alloys usually exhibit poor formability at room temperature, because of their hexagonal close-packed (HCP) structures and the limited number of slip systems, which restricts large-scale applications. It is well known (Muransky et al., 2009; Proust et al., 2009) that there are two easy deformation systems in Mg alloys: basal <a> slip and tensile twinning (or extension twinning) $\{10.2\}<10.1\rangle$. Since twinning is a unidirectional deformation mechanism, it only produces either tensile or compressive strains along the crystallographic c-axis in HCP metals, depending on the $c / a$ ratio. Because the $c / a$ axial ratio of $\mathrm{Mg}$ is $1.624<\sqrt{3}$, the most prominent twinning in HCP Mg alloys is $\{10.2\}<10.1>$ tensile twinning (Brown et al., 2005b), involving shearing along the $\{10.2\}$ planes and $\langle 10.1>$ directions. The tensile twinning results in a tensile strain parallel to the c-axis or a compression strain perpendicular to the c-axis (Christian and Mahajan, 1995; Agnew et al., 2002; Barnett, 2007; Wu et al., 2008b). It is essential to study the tensile twinning in wrought $\mathrm{Mg}$ alloys, since the tensile twinning provides a strain along c-axis, while the other easy slip system, the basal <a> slip, under a certain loading condition (such as inplane compression or through-thickness tension), fails to do so. 
The comprehensive research (Kim et al., 2005; Ishihara et al., 2007; Jiang et al., 2007; Jain et al., 2008; Wu et al., 2010; Khan et al., 2011; Ma et al., 2012; Yu et al., 2012; El Kadiri et al., 2013; Khosravani et al., 2013; ; Liu et al., 2013; Stanford and Barnett, 2013) concentrated on the deformation mechanism of wrought $\mathrm{Mg}$ alloys by employing a mechanical-testing method combined with in-situ or ex-situ optical microscopy (OM), scanning electron microscopy (SEM), electron backscattering diffraction (EBSD), transmission electron microscopy (TEM) techniques, etc. However, the information retrieved from the OM, SEM, EBSD, and TEM are constrained to the sample surface or thin film. Previous research also used in-situ neutron diffraction (Withers and Clarke, 1998; Gharghouri et al., 1999; Brown et al., 2005a; Brown et al., 2005b; Davies et al., 2005; Clausen et al., 2008; Muranskya et al., 2008; Woo et al., 2008; Hao et al., 2009; Muransky et al., 2009; Muransky et al., 2010; Abdolvand and Daymond, 2012; Balogh et al., 2012; Brown et al., 2013a; Brown et al., 2013b; Muransky et al., 2013; Prakash et al., 2013; Skippon et al., 2013;) to investigate the deformation mechanisms of Mg alloys and other HCPstructured materials during discontinuous step-loading. The different dominant deformation modes were successfully distinguished from the perspective of diffraction, when Mg alloys were subjected to the initial texture and strain-path changes. However, since step-loading is associated with a discontinuous measurement and a limited number of measurements, it is impractical to capture the exact moment of the transition of deformation modes, e.g., the transition from a twinning to dislocation deformation mode in wrought Mg alloys. The knowledge gap regarding the plastic deformation mechanism of Mg alloys still remains.

The theoretical models have been developed in the last two decades to simulate the deformation behavior of HCP-structured metals (Molinari et al., 1987; Lebensohn and Tome, 1993; Turner and Tome, 1994; Hama et al., 2013; Knezevic et al., 2013; Herrera-Solaz et al., 2014; Liu and 
Wei, 2014; Wang et al., 2014). Among them, the elastic-viscoplastic self-consistent (EVPSC) polycrystal model has been used to mimic the large strain behavior of $\mathrm{Mg}$ alloys under different deformation processes (Wang et al., 2010a; Wang et al., 2010b; Wang et al., 2011; Wang et al., 2012b). Very recently, the newly-developed twinning and de-twinning (TDT) model was implemented into the EVPSC model, called the EVPSC-TDT model, which has been proposed to describe the twinning and de-twinning behavior for HCP-structured polycrystalline materials and successfully applied to a Mg alloy AZ31B rolling sheet under cyclic loadings and strain-path changes (Wang et al., 2012a; Wang et al., 2013a), which can accurately account for the latticestrain evolution of interested $h \mathrm{kl}$ s during twinning and detwinning deformation process.

In the present research, the deformation dynamics and the effect of deformation history on plastic deformation in a wrought AZ31B Mg alloy have been studied using the state-of-the-art real-time in-situ neutron diffraction measurements under a continuous-loading condition, and the EVPSC-TDT model has been employed to quantify the deformation dynamics from a mechanistic point of view during strain-path changes. 


\section{Experimental procedures}

\subsection{Materials and mechanical testing}

A commercial AZ31B Mg rolled plate [a nominal composition 3.0 weight percent (w.t.\%) Al, 1.0 w.t. $\% \mathrm{Zn}$, and $\mathrm{Mg}$ as balance] in $\mathrm{H} 24$ temper (strain hardened and partially annealed) with 76 mm thickness was chosen. In rolled Mg alloys, a typical rolling texture can be expected (Wu et al., 2012) with the c-axes of the majority grains aligning to the plate-normal direction (ND), and perpendicular to the rolling direction (RD). It has been established that tensile twinning can be activated during in-plane compression or through-thickness tension (Agnew and Duygulu, 2005; Lou et al., 2007). Therefore, by tailoring the samples with the applied loading axis relative to the texture orientation, it is possible to manipulate the deformation behavior by changing the strain paths. For instance, by introducing a stress state, where tensile twinning, $\{10.2\}<10.1\rangle$, is the only dominant deformation mode, the deformation mechanism of tensile twinning can be isolated for the investigation. Thus, cylindrical dog-bone specimens were machined with their axial direction aligned along RD of the plate. The sample gage section was $16 \mathrm{~mm}$ in length and $8 \mathrm{~mm}$ in diameter, according to the American Society for Testing and Materials (ASTM)

Standard E606-04. Prior to mechanical testing, the specimens were annealed at $345^{\circ} \mathrm{C}$ for 2 hours to remove the residual stress due to manufacturing and machining. The average grain size of the annealed AZ31B Mg alloy was approximately $40 \mu \mathrm{m}$. The pole figure of the annealed sample has been published elsewhere (Wu et al., 2012), which is also used as the input of the initial texture for the simulations in the current study.

2.2 Neutron diffraction measurements and data analysis 
The real-time in-situ neutron diffraction measurements were performed under a continuousloading condition using the state-of-the-art VULCAN Engineering Diffractometor at the Spallation Neutron Source (SNS), Oak Ridge National Laboratory (ORNL) (Wang et al., 2006; Wang and Stoica, 2009; Wang et al., 2010d; An et al., 2011). The schematic setup of the realtime in-situ neutron diffraction measurements (Wu et al., 2013) is presented in Fig. 1. Briefly, the specimen was mounted in the VULCAN loadframe horizontally and carefully aligned, so that the axial and horizontal-radial directions parallel to the $\mathrm{RD}$ and ND, respectively. The angle between the incident neutron beam and the sample was $45^{\circ}$. The two stationary detector banks, which located $\pm 90^{\circ}$ to the incoming beam, recorded simultaneously two complete diffraction patterns in the axial (RD of the rolling plate) and horizontal-radial (ND of the rolling plate) directions with diffraction vectors parallel $(\mathrm{Q} \|)$ and perpendicular $(\mathrm{Q} \perp)$ to the applied load, respectively. The neutron beam size was defined as $5 \mathrm{~mm}$ (horizontal) x $5 \mathrm{~mm}$ (vertical), and the 5 -mm receiving collimators were selected, leading to a neutron gauge volume of total $125 \mathrm{~mm}^{3}$. It is worthy to mention that approximately 1.9 million grains (the grain size is $40 \mu \mathrm{m}$, and the neutron gauge volume is $125 \mathrm{~mm}^{3}$ ) were measured during deformation, which suggests an excellent counting statistics for the neutron diffraction experiments.

During real-time in-situ neutron diffraction measurements, a specimen, designated as the "asreceived sample", was compressed to $-7 \%$ and followed by a reverse tension until failure. Another sample, denoted as the "pre-deformed sample", was deformed to 7\% strain in tension, and then applied with the same strain path as that of the as-received sample (after being unloaded to $0 \mathrm{MPa}$, deformed $7 \%$ in compression - unloaded to $0 \mathrm{MPa}$ - deformed $7 \%$ in tension). Both samples were loaded under a constant strain rate, $1.3 \times 10^{-5} \mathrm{~s}^{-1}$, under a strain control mode at room temperature. 


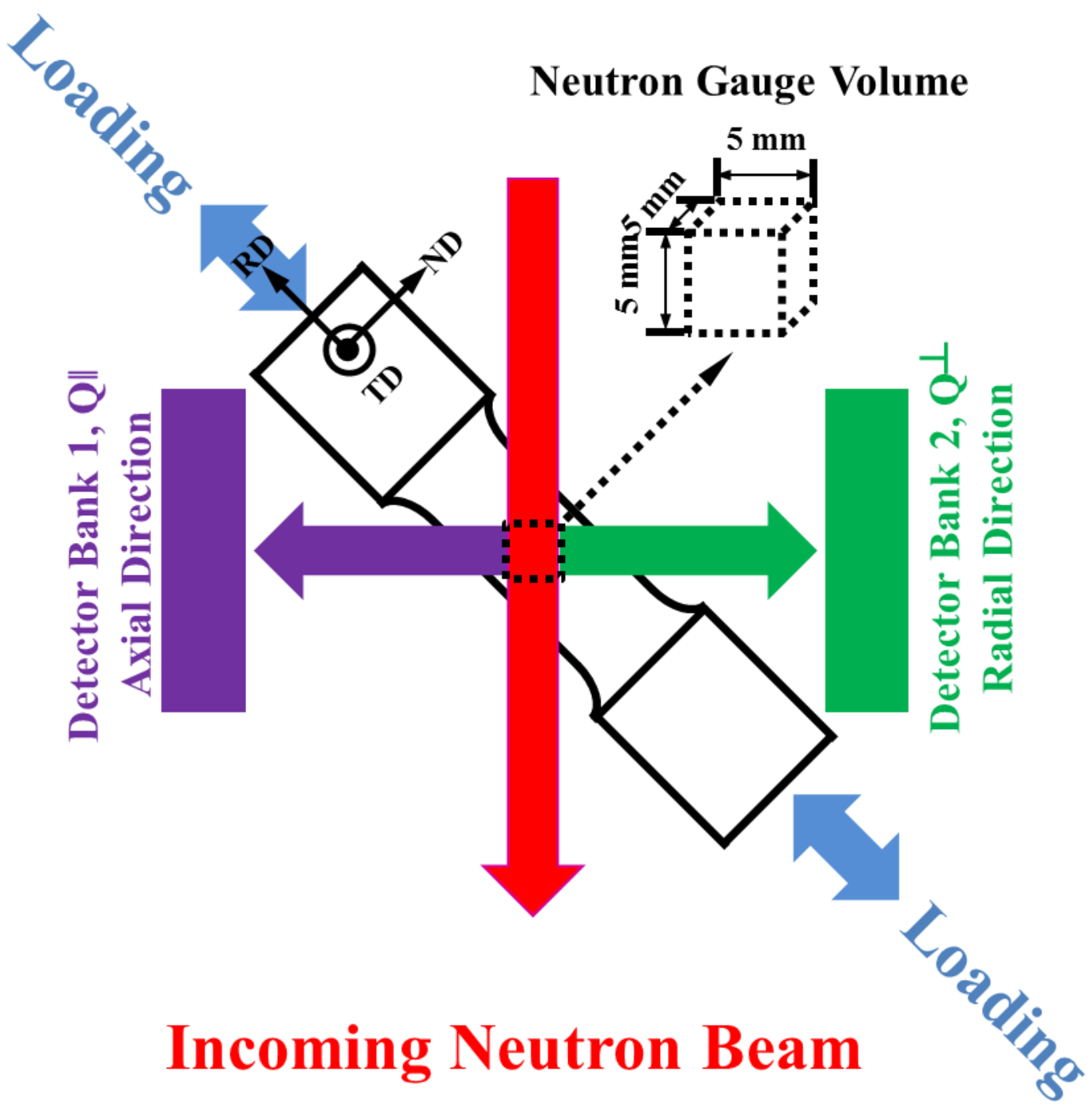

Figure 1 Schematic of a real-time in-situ neutron diffraction setup at VULCAN, SNS, ORNL (Wu et al., 2013). 
The real-time in-situ neutron diffraction measurements were performed under a continuous loading condition, which essentially eliminates the strain flow or the stress relaxation under the stress or strain control due to the holding (An et al., 2011; Wu et al., 2013). Neutron diffraction data were recorded successively during loading, and the data were chopped into small time bins afterwards using the event-based data reduction software, ULCAN Data Reduction and Interactive Visualization softwarE (VDRIVE) (An, 2012). Short time interval was determined, considering both the statistics and the quality of different patterns for data fitting, in order to obtain an adequate representative of the actual data. Neutron diffraction data were sliced every two minutes in the current study and synchronized with the loadframe information, such as the averaged load/stress and displacement/strain over two minutes. It is worth mentioning that because a small strain interval $\left(1.3 \times 10^{-5} \mathrm{~s}^{-1} \times 120 \mathrm{~s}=1.56 \times 10^{-3}\right)$ was chosen in the current study, the average stress changes over every two minutes are thought to be acceptable. The stress interval from point to point varies from $2 \mathrm{MPa}$ to $8 \mathrm{MPa}$ in the plastic-deformation regions. The neutron diffraction peak intensities of different $h k l s$ after the background subtraction were normalized to the incoming beam energy. The lattice strains of different $h k l$ s were calculated, based on the equation below:

$$
\varepsilon^{h k l}=\frac{d^{h k l}-d_{0}^{h k l}}{d_{0}^{h k l}}
$$

where $\varepsilon^{h k l}$ is the lattice strain, $d_{0}^{h k l}$ and $d^{h k l}$ are the d-spacings before and after deformation, respectively. The counting time for the measurement of the reference at zero stress in both samples was 10 minutes, which is longer than the chopped data, in order to minimize the propagated $d_{0}^{h k l}$ statistical error. 
In Fig. 2, the two-dimensional (2-D) real-time in-situ neutron diffraction results of the measured d range are plotted to reveal the deformation dynamics (the evolutions of diffraction peak intensities, d-spacings, and diffraction peak widths of different $h k l s$ ) during strain-path changes for the as-received and pre-deformed samples in both axial and radial directions. The microscopic response during deformation, such as simultaneous diffraction peak position, intensity, and width changes in different groups of grains, can be retrieved. For example, as shown in Fig. 2(a), the diffraction peak intensity of (00.2) grains in the as-received sample experiences appearing-disappearing sequence during compression-reverse tension, which suggests the twinning-detwinning behavior. From this plot, the moment when the twinning and detwinning starts and ends can be readily determined. The detailed description of neutron diffraction results will be provided in the Results and Discussion sections. 

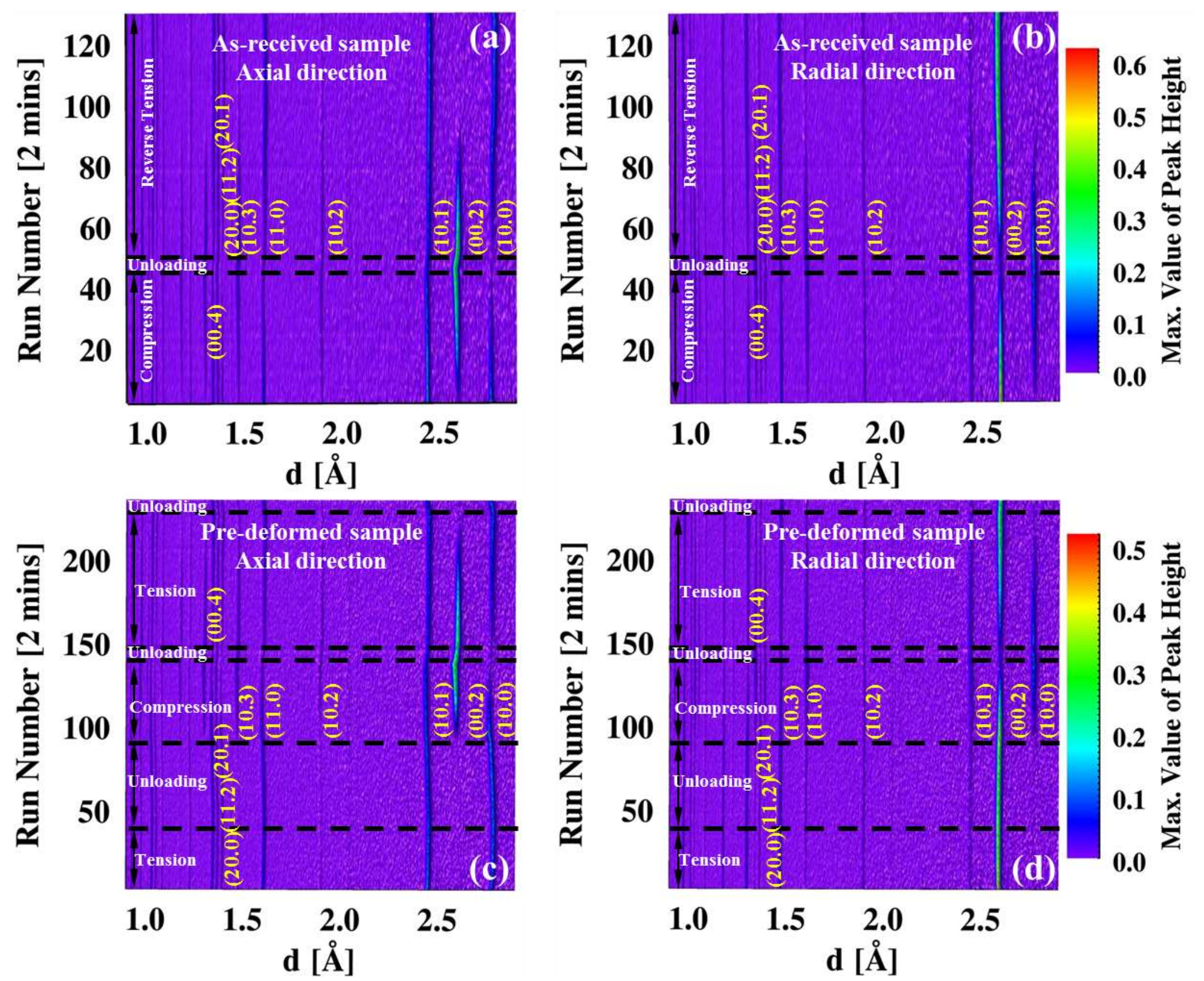

Figure 2 The two-dimensional (2-D) real-time in-situ neutron diffraction results of the measured d range during strain-path changes for: (a) the as-received sample in the axial direction, (b) the as-received sample in the radial direction, (c) the pre-deformed sample in the axial direction, and (d) the pre-deformed sample in the radial direction. The color bars represent the maximum height of diffraction peaks. 


\section{Theory/Calculation}

\subsection{The EVPSC constitutive model}

The recently proposed physics-based crystal plasticity model, the EVPSC-TDT model (Wang et al., 2012a; Wang et al., 2013a), developed by implementing the TDT model into the EVPSC model (Wang et al., 2010a; Wang et al., 2010c; Wang et al., 2011; Wang et al., 2013b), is well suitable to study the twinning and detwinning behavior of HCP polycrystals under complex loading conditions. In the current study, the EVPSC-TDT model is employed to study the deformation mechanism of the wrought AZ31B Mg alloy during strain-path changes.

In this section, we briefly recapitulate the EVPSC-TDT model, mainly for the purpose of definition and notation. For details, we refer to Wang et al. (Wang et al., 2010c; Wang et al., 2013b). The EVPSC model developed by Wang et al. (Wang et al., 2010c) for polycrystalline materials is a completely general elastic visco-plastic, fully anisotropic, self-consistent polycrystal model, applicable to large strains and to any crystal symmetry. It has been demonstrated that the EVPSC model can be used to simulate the large strain behavior of HCP polycrystalline materials under various deformation processes (Wang et al., 2010a; Wang et al., 2010b; Wang et al., 2011; Wang et al., 2012b). Very recently, a new constitutive model to describe twinning-detwinning behavior for polycrystalline materials with the HCP crystallographic structure has been developed (Wang et al., 2012a; Wang et al., 2013b) and implemented in the EVPSC polycrystal plasticity mode. The TDT model has been applied to Mg alloy AZ31B sheet under cyclic loadings and strain path changes as well as large strain torsion (Wang et al., 2012a; Guo et al., 2013; Wang et al., 2013a; Wang et al., 2013b). It has been demonstrated that the new twinning model is able to capture key features associated with the experimentally observed twinning and detwinning behaviors. 
The plastic deformation of a crystal is assumed to resulte from crystallographic slip and twinning on crystallographic system $\left(\mathrm{s}^{\alpha}, \mathrm{n}^{\alpha}\right)$. Here, $\mathrm{s}^{\alpha}$ and $\mathrm{n}^{\alpha}$ are, respectively, the slip/twinning direction and the normal of the slip/twinning plane for system $\alpha$. In the present study, we assume the plastic deformation is due to three types of slip systems: Basal $\langle a\rangle$ $(\{0001\}<11 \overline{2} 0>)$, Prismatic $\langle a>(\{10 \overline{1} 0\}<11 \overline{2} 0>)$ and Pyramidal $\langle c+a>(\{\overline{1} \overline{1} 22\}<\overline{1} \overline{1} 23>$ ), and one twinning mode: the $\{10 \overline{1} 2\}<\overline{1} 011>$ tensile twin system. The plastic strain rate tensor for the crystal can be written as

$$
\mathrm{d}^{p}=\sum_{\alpha} \dot{\gamma}^{\alpha} \mathrm{P}^{\alpha}
$$

in terms of the shear rate, $\dot{\gamma}^{\alpha}$, and the Schmid tensor, $P^{\alpha}=\left(s^{\alpha} n^{\alpha}+n^{\alpha} s^{\alpha}\right) / 2$ for system $\alpha$. Regardless of slip or twinning, the driving force for shear rate, $\dot{\gamma}^{\alpha}$, is the resolved shear stress, $\tau^{\alpha}=\sigma: \mathrm{P}^{\alpha}$, where $\sigma$ is the Cauchy stress tensor. For slip,

$$
\dot{\gamma}^{\alpha}=\dot{\gamma}_{0}\left|\tau^{\alpha} / \tau_{c r}^{\alpha}\right|^{\frac{1}{m}} \operatorname{sgn}\left(\tau^{\alpha}\right)
$$

and for twinning,

$$
\dot{\gamma}^{\alpha}=\left\{\begin{array}{cc}
\dot{\gamma}_{0}\left|\tau^{\alpha} / \tau_{c r}^{\alpha}\right|^{\frac{1}{m}} & \tau^{\alpha}>0 \\
0 & \tau^{\alpha} \leq 0
\end{array}\right.
$$

where $\dot{\gamma}_{0}$ is a reference shear rate, $\tau_{c r}^{\alpha}$ is the critical resolved shear stress (CRSS), and $m$ is the strain-rate sensitivity.

For both slip and twinning, the evolution of CRSS, $\tau_{c r}^{\alpha}$, is given by: 


$$
\dot{\tau}_{c r}^{\alpha}=\frac{d \hat{\tau}^{\alpha}}{d \Gamma} \sum_{\chi} h^{\alpha \chi}\left|\dot{\gamma}^{\chi}\right|
$$

where $\Gamma=\sum_{\beta} \int\left|\dot{\gamma}^{\alpha}\right| d t$ is the accumulated shear strain in the grain, and $h^{\alpha \chi}$ are the latent hardening coupling coefficients, which empirically account for the obstacles on system $\alpha$ associated with system $\chi . \hat{\tau}^{\alpha}$ is the threshold stress and is characterized by:

$$
\hat{\tau}^{\alpha}=\tau_{0}^{\alpha}+\left(\tau_{1}^{\alpha}+h_{1}^{\alpha} \Gamma\right)\left(1-\exp \left(-\frac{h_{0}^{\alpha}}{\tau_{1}^{\alpha}} \Gamma\right)\right)
$$

Here, $\tau_{0}, h_{0}, h_{1}$, and $\tau_{0}+\tau_{1}$ are the initial CRSS, the initial hardening rate, the asymptotic hardening rate, and the back-extrapolated CRSS, respectively.

Because it is rare that a grain can be fully twinned, a threshold twin volume fraction is defined in the model to terminate twinning. Consequently, the TDT model introduces two statistical variables: accumulated twin fraction, $V^{a c c}$, and effective twinned fraction, $V^{\text {eff }}$. More specifically, $V^{a c c}$ and $V^{\text {eff }}$ are the weighted volume fraction of the twinned region and volume fraction of twin terminated grains, respectively. The threshold volume fraction, $V^{\text {th }}$, is defined as

$$
V^{t h}=\min \left(1.0, A_{1}+A_{2} \frac{V^{e f f}}{V^{a c c}}\right)
$$

where $A_{1}$ and $A_{2}$ are two material constants for numerically controlling the observed exhaustion of twinning. Roughly speaking, $A_{1}$ controls the length of the strain-hardening plateau, and $A_{2}$ controls the increased rate of strain hardening at the end of the plateau. While the type of behavior is generally understood to be the case, a complete physical explanation of why grains 
undergo this trend in the twin exhaustion behavior, rather than continue to twin to $100 \%$ is not yet known. 


\section{Results}

\subsection{Macroscopic behavior}

Figure 3 shows the measured and predicted monotonic uniaxial tension and compression stressstrain curves of the as-received material along the RD. The monotonic tension and compression tests were at a strain rate of $\dot{\varepsilon}=10^{-4}$. In the simulation results shown in Fig. 3, the roomtemperature elastic constants of the $\mathrm{Mg}$ single crystal are $C_{11}=58.0, C_{12}=25.0, C_{13}=20.8$, $C_{33}=61.2$ and $C_{44}=16.6(\mathrm{GPa})$. The reference slip/twinning rate, $\dot{\gamma}_{0}$, and the rate sensitivity, $m$, are prescribed to be the same for all slip/twinning systems: $\dot{\gamma}_{0}=0.001 \mathrm{~s}^{-1}$ and $m=0.05$, respectively. Values of the hardening parameters are estimated by fitting numerical simulations of monotonic uniaxial tension and compression along the RD to the corresponding experimental flow curves. It is worth mentioning that it is assumed that all slip systems and tensile twinning harden with one another at the same level for the sake of simplicity, thus all the latent hardening parameters, $h^{s t}$, are designated as 1 in the current study. From Fig. 3a, it is found that the EVPSC-TDT model can well reproduce the experimental curves of the as-received material, by using a single set of hardening parameters. The values of these parameters are listed in Table 1 and will be used in all the simulations reported in the present paper. The characteristics of the stress and strain curves (Fig. 3a) and the relative activities of slip/twinning under uniaxial tension and compression along the RD (Fig. 3b) clearly reveal the importance of twinning in compression along the $\mathrm{RD}$, while the uniaxial tension along the $\mathrm{RD}$ is mainly governed by the crystallographic slips. More specifically, the model predicts that mostly basal and prismatic <a> slips accommodate the plastic deformation under uniaxial tension. Under uniaxial compression, at small strains, tensile twinning is very active, most grains reorient their c-axis along the RD, 
and the remaining plasticity is accommodated by basal slip. When the tensile twining activity is dramatically reduced, at a strain of $\varepsilon \approx 5 \%$, the model shows a significant increase in basal slip activity and a noticeable increase in pyramidal $\langle c+a\rangle$ slip activity. It is worth mentioning that we took a traditional approach to use only two experiments to completely determine all the material parameters involved in the model. The values of the parameters are in a realistic range typically found for the material.
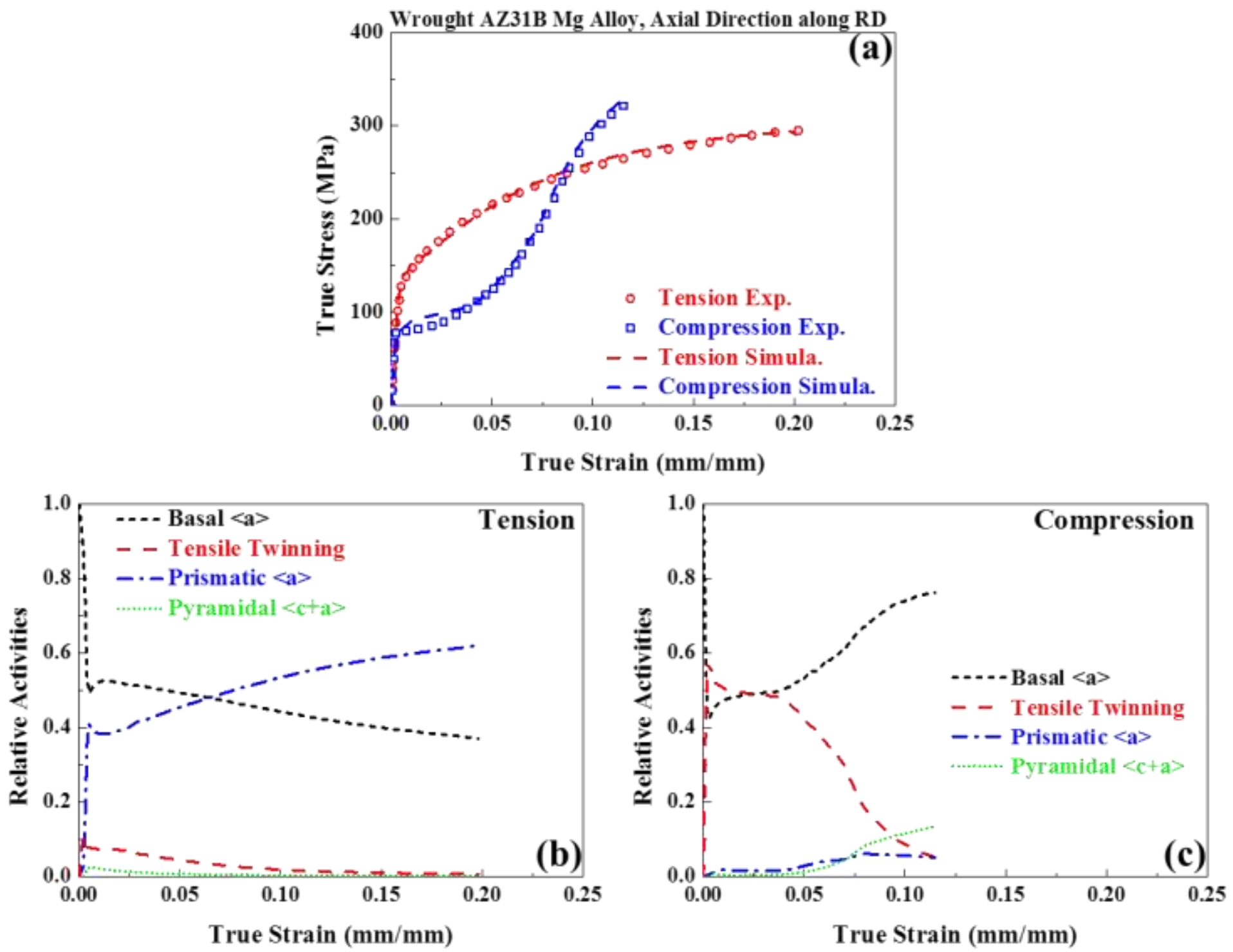

Figure 3 Monotonic uniaxial tension and compression of the as-received material along the RD. (a) Measured (open symbols) and fitted stress-strain curves (dashed lines); (b) Relative activities of various slip and twinning mechanisms. 
Table 1 List of material parameters for slip and twin systems used in the EVPSC-TDT model. The parameter, $h^{s t}$, lists latent hardening of the twinning activity upon other slip modes. All other latent-hardening parameters are 1.

\begin{tabular}{cccccccc}
\hline Mode & $\boldsymbol{\tau}_{\mathbf{1}}(\mathrm{MPa})$ & $\boldsymbol{\tau}_{\mathbf{2}}(\mathrm{MPa})$ & $\mathbf{h}_{\mathbf{0}}(\mathrm{MPa})$ & $\mathbf{h}_{\mathbf{1}}(\mathrm{MPa})$ & $\mathbf{h}^{\text {st }}$ & $\mathbf{A}_{\mathbf{1}}$ & $\mathbf{A}_{\mathbf{2}}$ \\
\hline Basal & 12 & 1 & 10 & 0 & 1 & \\
Prismatic & 78 & 55 & 600 & 30 & 1 & \\
Pyramidal & 100 & 140 & 2500 & 40 & 1 & & \\
Extension twin & 35 & 10 & 100 & 10 & 1 & 0.65 & 0.75 \\
\hline
\end{tabular}

Figure 4 demonstrates the mechanical-testing and simulation results during the aforementioned strain-control profile for both the as-received and pre-deformed samples (Wu et al., 2013). The orange arrows and circled numbers marked in Fig. 4 identify the loading paths. The open symbols represent the experimental results, and the dashed lines stand for the simulation results. The mechanical tests were performed at a constant strain rate, $\dot{\varepsilon}=1.3 \times 10^{-5} \mathrm{~s}^{-1}$. Note that the strain rate under monotonic uniaxial tension and compression shown in Fig. 3 is $\dot{\varepsilon}=10^{-4} \mathrm{~s}^{-1}$.

As presented in Fig. 4(a), the as-received sample was compressed to - 7\% strains, then unloaded to $0 \mathrm{~N}$, followed by the reverse tension until sample failure. During compression, relatively low compressive yield strength was observed, followed by a gradual increase of stresses until approximately - $103 \mathrm{MPa}$ (marked as a red dot). Subsequently, the slope of the stress-strain curve started to increase, suggesting strain hardening. When the sample was unloaded to $0 \mathrm{~N}$, the material exhibited the pseudoelastic behavior, which, in general, is defined as nonlinearity during unloading. In the reverse tension, relative low yield strength was distinguished, followed by slight strain hardening until the stress reached $118 \mathrm{MPa}$ (marked as a green dot). From approximately $118 \mathrm{MPa}$ to $217 \mathrm{MPa}$ (marked as a blue dot), the stress increased steeply with 
increasing strain. When the sample was further stretched, the stress-strain curve tended to be flat. Based on the work-hardening rate (the slope of the stress-strain curve) variation, the stress-strain curve in Fig. 4 can be divided into several stress ranges, marked as uppercase and lowercase Roman numerals for as-received and pre-deformed samples, respectively.

The pre-deformed sample [in Fig. 4(b)] has a similar trend as the as-received sample [in Fig. 4(a)]. However, an obvious increase in the compressive yield strength, approximately $40 \mathrm{MPa}$, was observed. Moreover, the pre-deformed sample showed apparent strengthening during compression, due to the work hardening from the prior in-plane tension. In the following reverse tension, the effect of pre-tension became less significant, and only a small increase of the tensile yield strength, about $13 \mathrm{MPa}$, was identified.

As mentioned previously, only monotonic uniaxial tension and compression tests were used to completely determine all the material parameters involved in the model. Furthermore, the TDT model does not introduce any additional parameters for detwinning. Therefore, the calculated stress and strain curves shown in Fig. 4 are predictions. It is clear that the EVPSC-TDT model can well reproduce the experimental curves. 


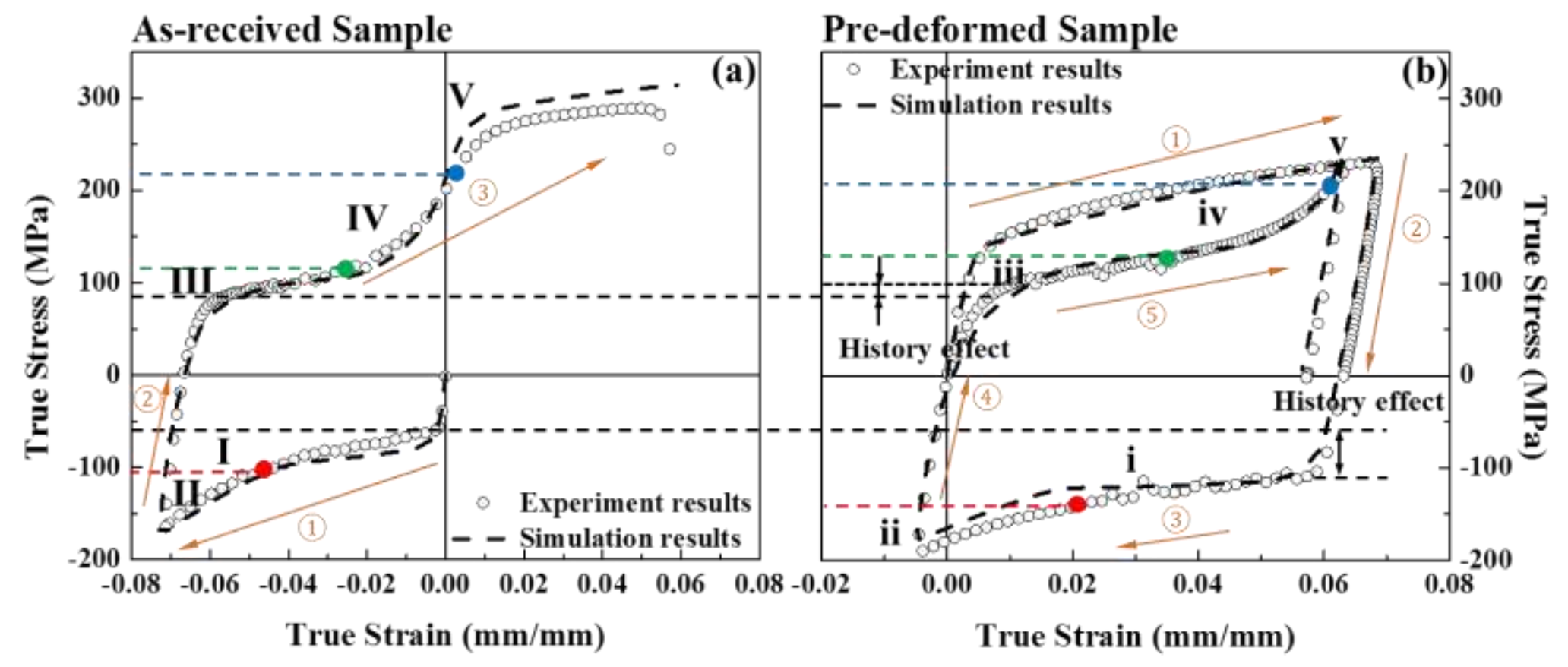

Figure 4 The stress vs. strain curves during strain-path changes for: (a) the as-received sample and (b) the pre-deformed sample (Wu et al., 2013). The loading sequence was marked by the orange arrows and circled numbers. The inflection points of the stress-strain curves were marked by colored dots. 


\subsection{Microscopic response}

\subsubsection{Diffraction peak intensity evolution}

Figure 5 presents the diffraction peak intensity evolution in different $h k l s$ in the axial direction from the real-time in-situ neutron diffraction measurements and simulations for both the asreceived and pre-deformed samples. The separation of stress ranges in Fig. 5 is inherited from Fig. 4. Thus, the microscopic response can be isolated in each macroscopic stress range to examine.

For the as-received sample in Fig. 5(a), little variation on diffraction peak intensities for (10.0) and (11.0) grains was observed in the elastic region during compression. The (00.2) diffraction peak intensity was absent until the stress exceeded the yield strength. In the stress range I, the diffraction peak intensities of (10.0) and (11.0) grains decreased dramatically, while the (00.2) diffraction peak intensity grew rapidly due to tensile twinning. When the compressive stress went into the stress range II, both the decrease of (10.0) peak intensity and the increase of (00.2) peak intensity decelerated simultaneously. During unloading, no apparent diffraction peak intensity variation was observed in (10.0), (00.2), and (11.0) grains, suggesting that no detwinning occurred. No evident detwinning process was identified even in the elastic region (below $87 \mathrm{MPa}$ ) during reverse tension, since the diffraction peak intensities of (10.0), (00.2), and (11.0) grains remained almost unchanged. In the stress range III, the diffraction peak intensities of (10.0) and (11.0) grains increased abruptly, and simultaneously, the diffraction peak intensity of (00.2) twin grains diminished significantly, suggesting that the detwinning process occurred intensively. In the stress range IV, the diffraction peak intensity of (00.2) twin grains decreased gradually, and the diffraction peak intensities of (10.0) and (11.0) detwinned 
grains increased slowly. Nearly all of the (10.0) and (11.0) diffraction peak intensities recovered, and the (00.2) twin grains disappeared, when the stress reached $217 \mathrm{MPa}$. The diffraction peak intensities of interested $h k l s$ stayed unchanged when the sample was further pulled to the stress range $\mathrm{V}$.

The diffraction peak intensity evolution of the pre-deformed sample demonstrated the analogous tendency to the as-received sample during both compression and reverse tension, as shown in Fig. 5(b). It is interesting that the stress rang from the activation to the exhaustion of the tensile twinning (stress range i) in the pre-deformed sample was narrower than the as-received sample (stress range I). Moreover, the duration of the detwinning process was shorter in the predeformed sample (stress range iii) than in the as-received sample (stress range III).
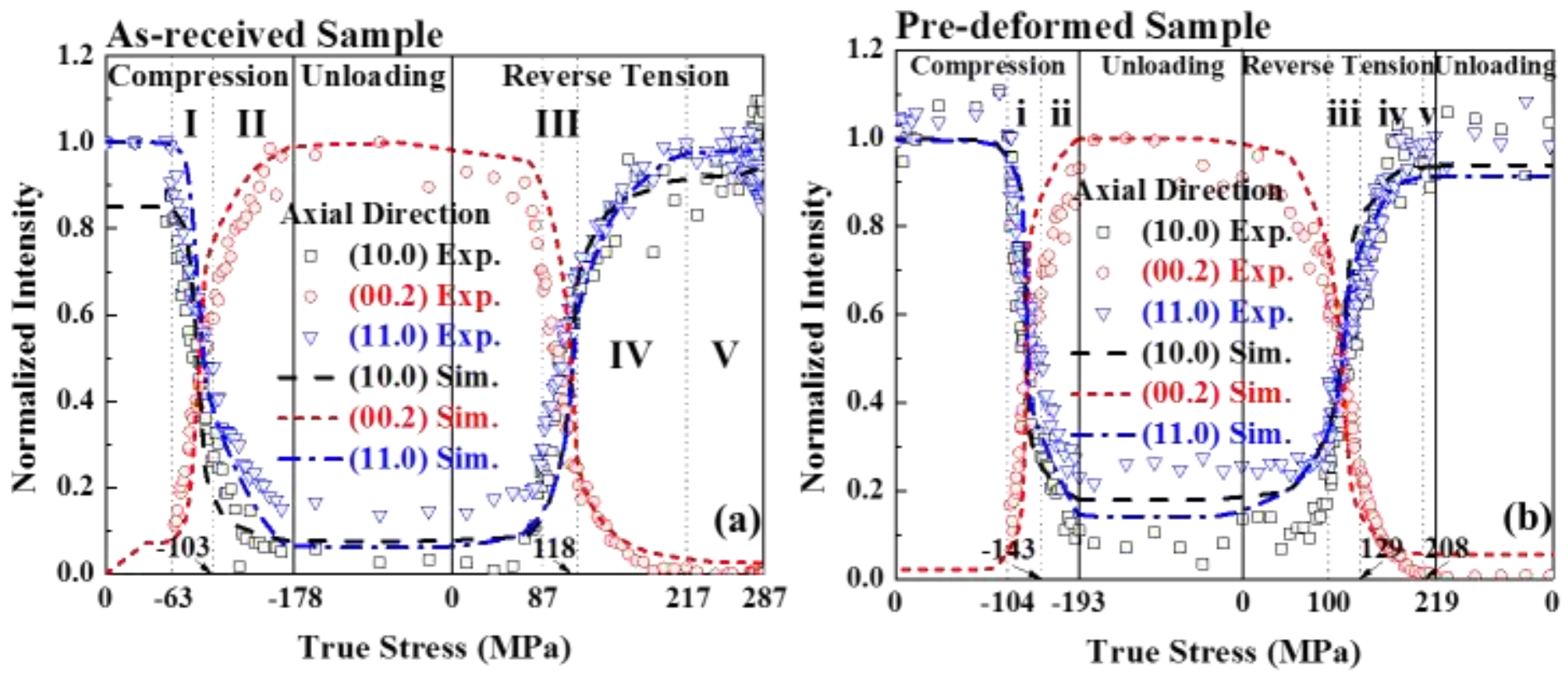

Figure 5 The diffraction peak intensity evolution of certain $h \mathrm{kls}$ as a function of stress in the axial direction from real-time in-situ neutron diffraction measurements (open symbols) and simulations (dashed lines) for: (a) the as-received sample and (b) the pre-deformed sample. 
To the best of the authors' knowledge, it is the very first time that the EVPSC-TDT model was employed to simulate the twinning and detwinning behavior under a continuous-loading condition during strain-path changes in a HCP-structured Mg alloy. It should be emphasized that the simulated diffraction peak intensity is proportional to the volume fraction of grains, which satisfy the Bragg's law and orientate within the coverage of the simulated detectors. In the current study, the simulated results were compared with the experimental data on a relative basis. In the simulations, the instantaneous intensities of the (10.0) and (11.0) grains along the axial direction were normalized by their corresponding initial values. However, for the (00.2) grains, the instantaneous intensity was normalized by its largest value, because the initial intensity was extremely small before deformation. The simulation results support the experimental observation by showing that no obvious diffraction peak intensity variation in (10.0), (00.2), and (11.0) grains during unloading, indicating that no detwinning occurs, which is contrary to previous studies (Muransky et al., 2009; Wu, 2009; Wu et al., 2008a; Wu et al., 2008b). The details will be provided in the Discussion.

\subsubsection{The lattice-strain evolution}

Figure 6(a) describes the relationships between the internal strain evolution and stress in the axial direction during compression for the as-received sample. In the stress range I, the lattice strain of (11.0) grains increased more rapidly than (10.0) and (10.1) grains. At the emergence of the (00.2) twins, the lattice strain of (00.2) grains increased at a similar rate as those in (10.0) and (10.1) grains, but slower than that in (11.0) grains. In the stress range II, the lattice strain of (11.0) and (10.1) grains increased slowly until saturated. Simultaneously, the internal strain was rapidly transferred to (00.2) twin grains, which, in the meantime, became the majority of the population among all orientation grains in the axial direction due to the tensile twinning, as presented in Fig. 
6(a). The final lattice strain in (00.2) twin grains reached 4,628 $\mu \varepsilon\left(10^{-6}\right.$ strain), which was higher than in other orientation grains. In the radial direction, the lattice strain of (00.2) grains saturated immediately at the compressive yield strength, $-63 \mathrm{MPa}$, as presented in Fig. 6(c).

The lattice-strain evolution of the pre-deformed sample in Fig. 6(b) in the axial direction during compression resembled the as-received sample in general. However, some differences can be noticed. First, the yield strength in the pre-deformed sample is much higher than the as-received sample. It should be noted that (10.0) and (11.0) grains contained a small amount of residual tensile strains at $0 \mathrm{MPa}$, while (10.1) grains had nearly zero residual strain in the pre-deformed sample. Second, at the same stress level, the lattice strain of (00.2) twin grains in the predeformed sample was much less than in the as-received sample. For example, at a stress level, $150 \mathrm{MPa}$, the lattice strain of (00.2) grains in the pre-deformed sample, 2,195 $\mu \varepsilon$, was much lower than the as-received sample, 4,256 $\mu \varepsilon$. Third, the lattice strain of (11.0) grains in the predeformed sample was the highest, compared with (10.0), (11.0), and (10.1) grains by the end of compression deformation, while the lattice strain of (00.2) grains was the highest in the asreceived sample. In Fig. 6(d), the (00.2) grains in the radial direction for the pre-deformed sample also yielded right after the stress reached the yield strength, as the as-received sample. However, a compressive residual strain in (00.2) grains was observed at $0 \mathrm{MPa}$, which was caused by the pre-deformation.

When the sample was unloaded to $0 \mathrm{MPa}$ after compression, the (10.1) grains exhibited a small amount of tensile residual strains in the as-received sample in the axial direction, while the compressive residual strains can be seen in (11.0) grains and (00.2) twin grains, as shown in Fig. 6(e). It should be emphasized that (00.2) twin grains contain a relatively large compressive residual strain, about $1,067 \mu \varepsilon$. Because a large amount of a compressive lattice strain $(4,628 \mu \varepsilon)$ 
was accumulated in the (00.2) twin grains during compression, as shown in Fig. 6(a), a certain amount of compressive residual strains was remained after rapid unloading. In Fig. 6(f), it is obvious that as the major population, the (00.2) twin grains had a very small tensile residual strain in the pre-deformed sample. Moreover, the (10.1) tensile residual strain in the predeformed sample was higher than the as-received sample.

Figure $6(\mathrm{~g})$ demonstrates the internal-strain evolution of certain $h k l$ s during reverse tension in the axial direction for the as-received sample. In the stress range III, the lattice strain of (11.0) detwinned grains was higher than otherwise-oriented grains. The (00.2) twin grains started to yield, when the stress approached the stress range III, and completely saturated in the stress range IV. The lattice strains of (10.0) and (11.0) detwinned grains were enhanced rapidly in the stress ranges IV and V. In particular, the lattice strain was built up in (10.0) detwinned grains quickly. Generally, the lattice-strain evolution in the as-received and pre-deformed samples during reverse tension was similar. The difference lays in the rate of the lattice-strain increase in (10.0) detwinned grains. The rate of the lattice-strain increase in the pre-deformed sample was not as fast as in the as-received sample. 
It is noted that, in Fig. 6, the measured lattice strain evolutions are presented separately in order 
to interpret them in terms of the yielding and work-hardening behaviors in different deformation stages. Figure 7 shows the measured and predicted evolution of lattice strains in the axial direction during the entire deformation process for both the as-received and pre-strained samples. The agreement between the measured and predicted lattice strains is reasonably good. Although not shown in the figure, a similar observation could be made for the lattice strain evolution in radial direction. To our best knowledge, this is the very first time that the measured lattice strain
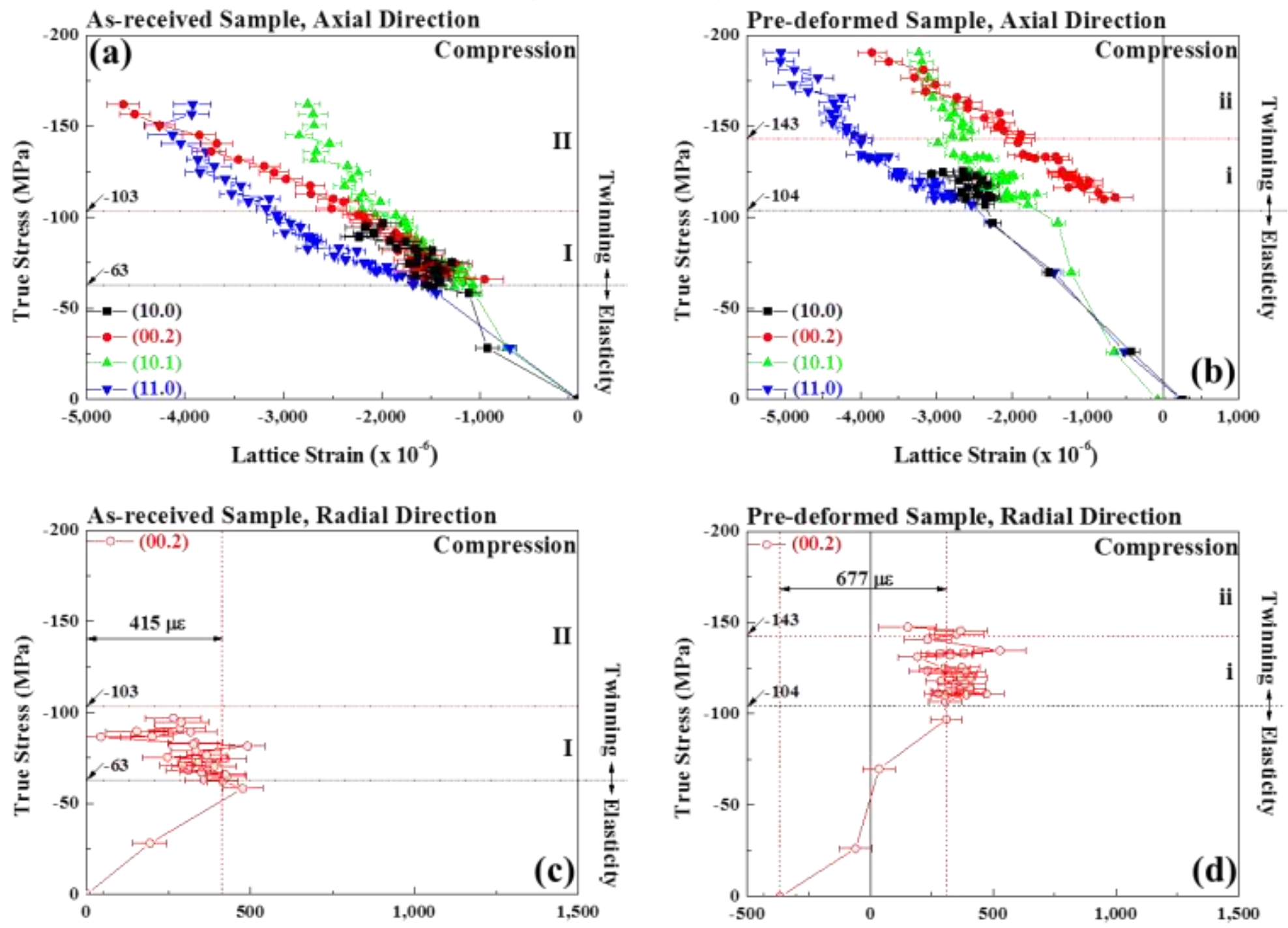

Lattice Strain $\left(x 1^{-5}\right)$

Lattice Strain $\left(\times 10^{-6}\right)$

evolution in a HCP-structured $\mathrm{Mg}$ alloy under reversed loading is

simulated. 

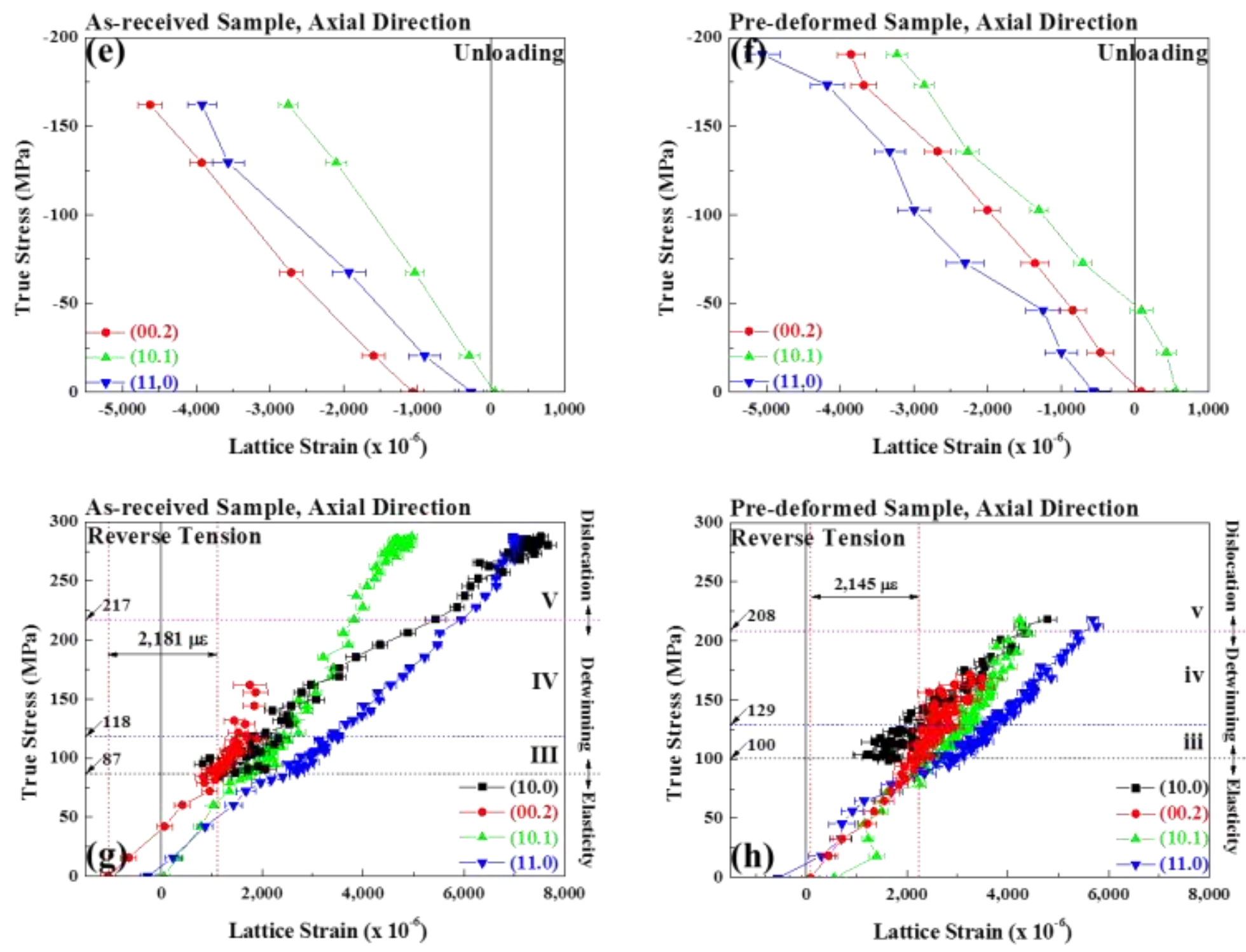

Figure 6 The internal-strain evolutions of certain $h k l$ s from real-time in-situ neutron diffraction measurements. (a), (c), (e), and (g) represent the lattice-strain evolution of the asreceived sample, and (b), (d), (f), and (h) are the lattice-strain evolution of the pre-deformed sample during strain-path changes. 

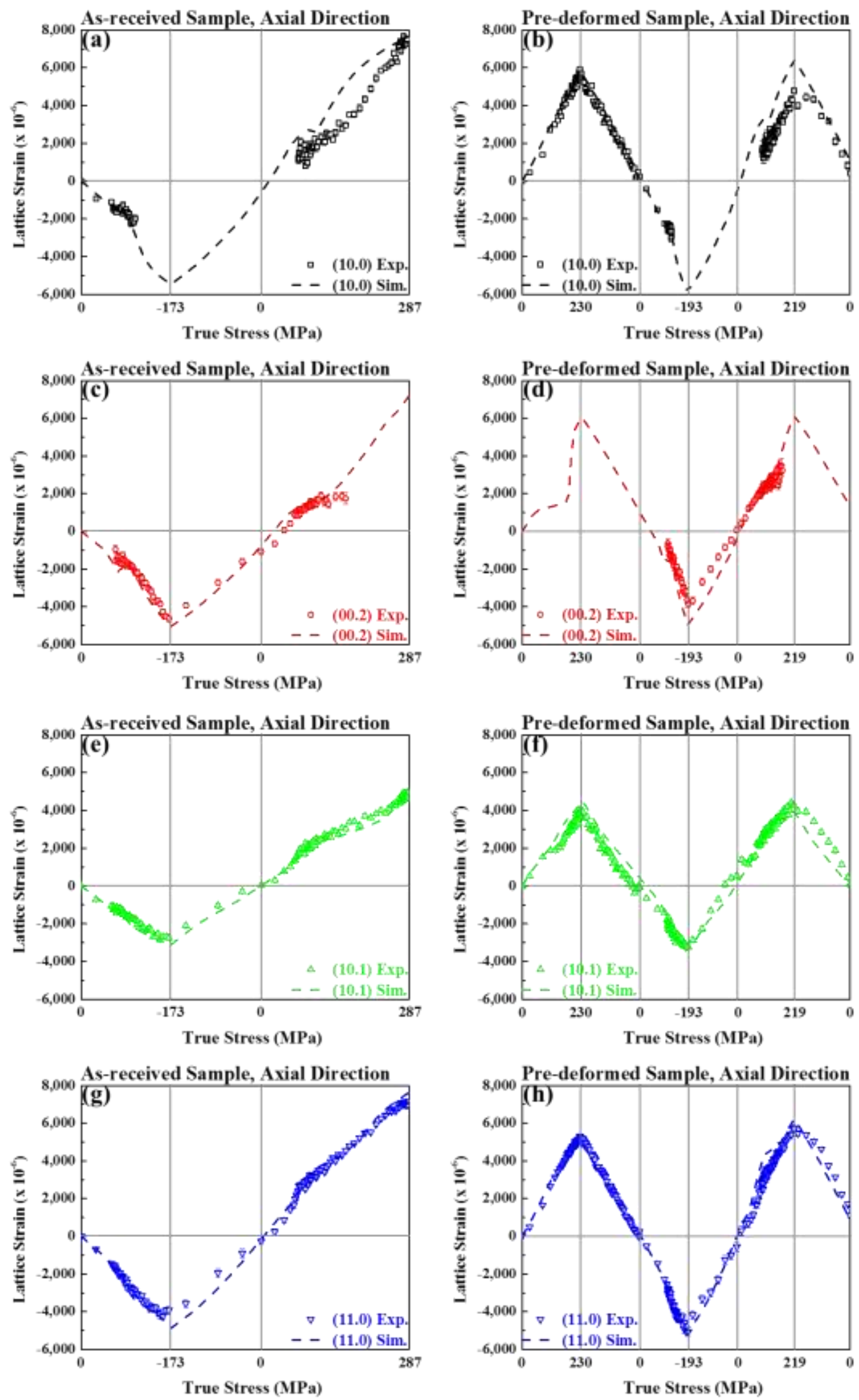

Figure 7. Measured (open symbols) and calculated (dashed lines) lattice strains in the axial direction as a function of applied stress for both the as-received (left) and pre-strained (right) samples. 


\section{Discussion}

5.1. Relationship between the macroscopic mechanical behavior and microscopic response

As presented in Fig. 4, the stress-strain curve can be divided into two sections in the plastic deformation region under compression for both the as-received and pre-deformed samples, based on the macroscopic-hardening-rate changes. Figure 5 illustrates the diffraction peak intensity evolution of certain $h k l s$ in the axial direction by the real-time in-situ neutron diffraction measurement and simulations for both samples. It is clear that in the stress ranges I and i, respectively, a slow and steady work hardening can be observed macroscopically for the asreceived and pre-deformed samples in Fig. 4. Microscopically, the diffraction peak intensities of (10.0) and (11.0) grains decrease significantly. Meanwhile, the diffraction peak intensity of (00.2) twin grains increases dramatically in Fig. 5. It is well known (Roberts, 1960) that a sudden re-orientation of grains of approximately $86^{\circ}$ occurs during tensile twinning-dominated deformation, owing to the $\mathrm{HCP}$ structure of $\mathrm{Mg}$, which results in the simultaneous variation in the diffraction peak intensity for (10.0) and (00.2) grains in one detector bank. It is also confirmed by the simulation results that the twin volume fraction, $f^{t w}$, enhances considerably in this stress range in Fig. 8, and the relative activity of tensile twinning becomes dominant in Fig. 9. Therefore, the stress range $\mathrm{I} / \mathrm{i}$ can be defined as the tensile twinning-dominant deformation region. In the stress range II/ii, rapid strain hardening can be observed in Figs. 3 and 4, while the rate of diffraction peak intensity changes in (10.0) and (00.2) grains decelerates in Fig. 5. This

trend indicates that the volume of tensile twinning keeps rising but tends to exhaust. In the meantime, the hard deformation mechanisms (i.e., prismatic $<a>$ or pyramidal $<\mathrm{c}+\mathrm{a}>$ dislocation slip) under the current loading condition are activated and become dominant in the twin grains (Wu et al., 2008a; Wu et al., 

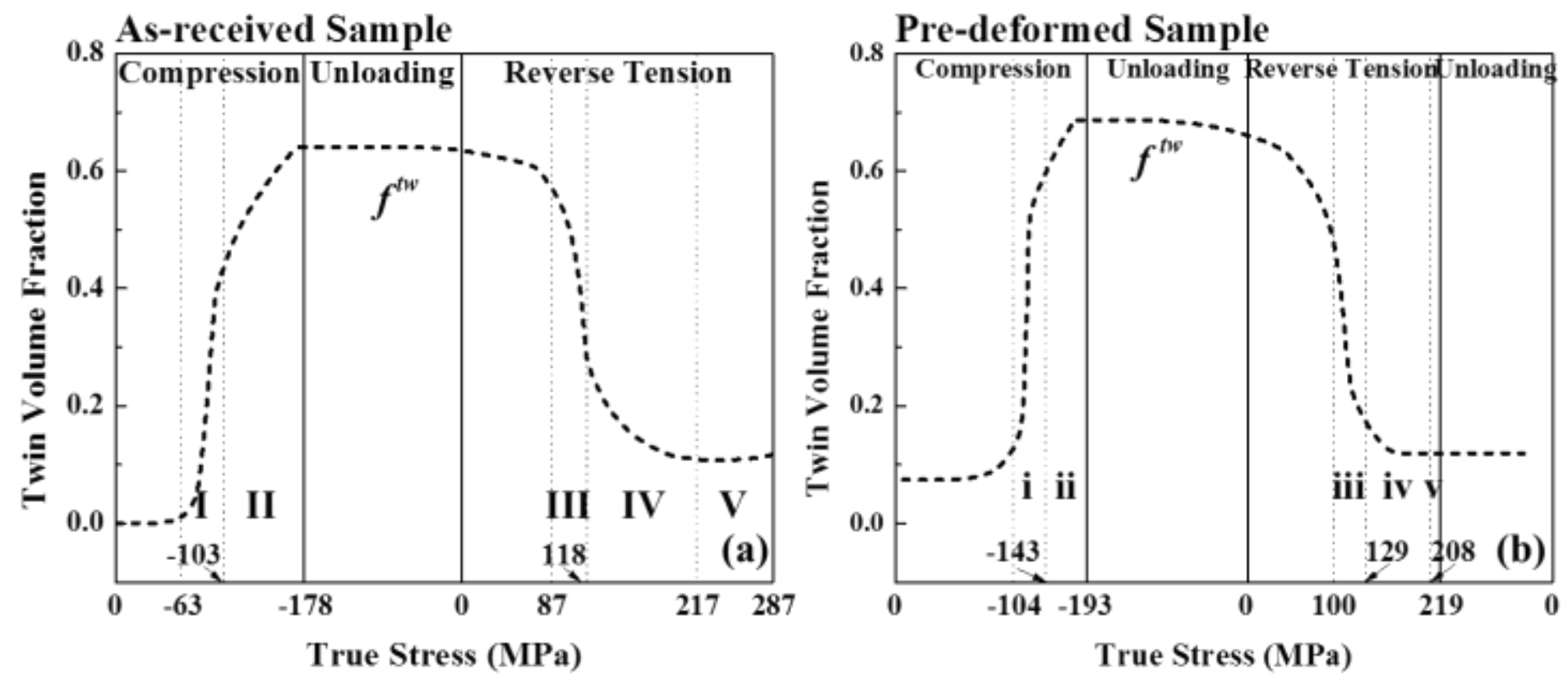

Figure 8 The simulation results of the twin-volume-fraction variation, $f^{t w}$, during strain-path changes for: (a) the as-received sample and (b) the pre-deformed sample.
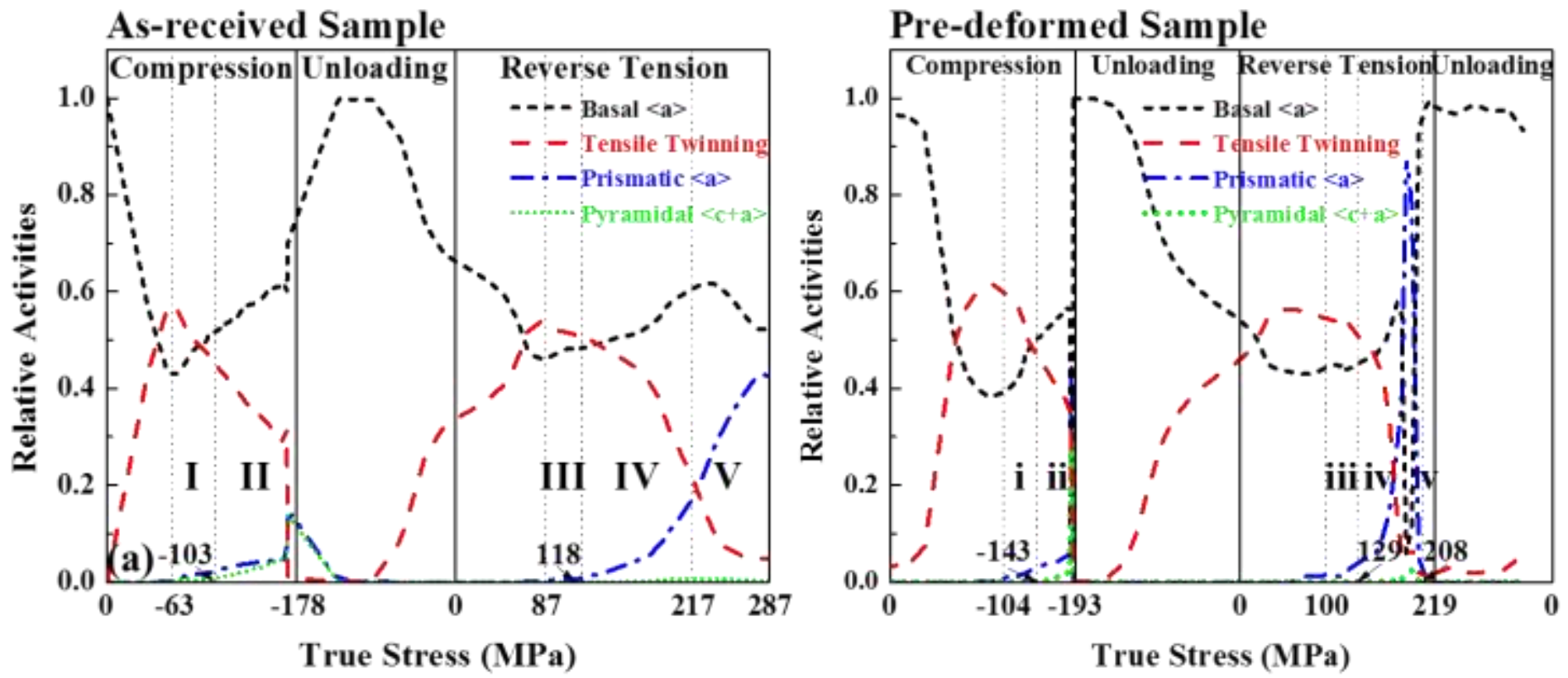

Figure 9 The relative activities of interested deformation modes during strain-path changes from the EVPSC-TDT model for: (a) the as-received sample and (b) the pre-deformed sample. 
2008b). The simulation results manifest that the rate of the twin-volume fraction increase tends to decline in this stress range, as displayed in Fig. 8. Moreover, the basal $<a>$ slip turns out to be dominant, while the prismatic $\langle\mathrm{a}\rangle$ and pyramidal $\langle\mathrm{c}+\mathrm{a}\rangle$ slips also become active, as demonstrated in Fig. 9. Consequently, the stress range II/ii can be characterized as the transition region from tensile twinning- to dislocation-dominated deformation. Similarly, during reverse tension, the stress ranges III/iii and IV/iv can be labeled as detwinning-dominated, and transition from detwinning- to dislocation-dominated deformation, respectively. In the stress range $\mathrm{V} / \mathrm{v}$, the diffraction peak intensities of (10.0), (00.2), and (11.0) grains cease changing in Fig. 5, so it can be tagged as the dislocation-dominated region.

The current study demonstrates that the relationship between the macroscopic mechanical behavior and microscopic response at a grain level can be clarified and elucidated by real-time in-situ neutron diffraction measurements. Moreover, the EVPSC-TDT model can be employed to quantify the deformation mechanisms during strain-path changes.

\subsection{The activation of the extension twinning}

It has been acknowledged that the Schmid law [Eq. (9)] is valid to calculate the onset of deformation twinning by assuming that the deformation twinning is governed by CRSS on the twin plane and in the twinning direction under a uniaxial loading condition (Gharghouri et al., 1999; Agnew et al., 2001; Agnew et al., 2003; Davies et al., 2005; Wu et al., 2008a; Wu et al., 2008b).

$$
\tau_{C R S S}=\sigma_{c} \cos \lambda \cos \chi
$$


where $\tau_{C R S S}$ is the CRSS, $\sigma_{c}$ is the critical applied stress, $\lambda$ and $\chi$ are the angles between the loading axis and twin plane normal and between the loading axis and the twinning direction, respectively. The lattice strain of (00.2) grains in the radial direction was used to calculate the onset of the activation of tensile twinning during compression. As marked in Figs. 6(c) and (d), the (00.2) grains began to yield, when the lattice strain reached 415 and $677 \mu \varepsilon$ for the asreceived and pre-deformed samples, respectively. Multiplying the Young's modulus of (00.2) for Mg (48 GPa) with lattice strain, the critical applied stress can be attained. Since the values of both $\lambda$ and $\chi$ equal approximately $45^{\circ}$, the CRSS of the as-received sample can be determined to be 10.0 MPa, which is somewhat lower than the results from previous studies (Agnew et al., 2001; Wu et al., 2008a; Wu et al., 2008b). The calculated CRSS of the pre-deformed sample is 16.2 $\mathrm{MPa}$, which is higher than the as-received sample. It suggests that the activation of tensile twinning in the pre-deformed sample is more difficult than the as-received sample.

It has been recognized that the onset of tensile twinning is governed by the CRSS. In the current study, the pre-deformed sample exhibits the higher CRSS for the activation of the tensile twinning than the as-received sample. It is believed that the high CRSS in the pre-deformed sample results from the intergranular residual strain/stress due to the deformation history. As shown in Fig. 6(d), the compressive intergranular residual strain was observed in (00.2) twin grains in the radial direction in the pre-deformed sample, which is caused by the predeformation. It is abnormal for $\mathrm{Mg}$, since the tensile intergranular residual strain was usually expected in the radial direction after tension deformation. A possible mechanism can be proposed that in order to activate tensile twinning, the compressive residual strain needs to be overcome in the pre-deformed sample, which leads to a high CRSS. 
The deformation-twinning mechanism can be categorized into two stages: twin nucleation and twin propagation. It is believed that the early stage of tensile twinning is governed by the twin nucleation (Yu et al., 2012), while the later stage is controlled by the twin propagation. By introducing a large amount of dislocations through in-plane tension in the current study, which serve as the twin nucleation sites, the twin nucleation process could be facilitated. Therefore, as long as the tensile twinning is activated, the twinning process could be accelerated. It agrees with the experimental observation in the present research that the shorter duration of twinning and detwinning process in the pre-deformed sample than the as-received sample.

\subsection{The detwinning process}

During unloading, the pseudoelastic behavior can be observed in the stress-strain curves for both samples, in Fig. 3. In previous studies, it was stated that the detwinning process occurred immediately upon unloading in wrought Mg alloys (Wu et al., 2008a; Wu et al., 2008b). The pseudoelastic behavior of wrought Mg alloys was attributed to the detwinning process previously

(Caceres et al., 2003; Muransky et al., 2009). In our previous paper (Wu et al., 2013), it is claimed that no detwinning was observed during unloading from the bulk average neutron diffraction results in both samples, since no apparent simultaneous diffraction peak intensity variation could be detected in (10.0) and (00.2) grains in the same detector bank. In the current paper, the simulation results indicate that the twin volume fraction, $f^{t w}$, keeps almost constant for both samples (Fig. 8), and the basal <a> slip rules over other deformation modes (Fig. 9) during unloading, which agrees well with the real-time in-situ neutron diffraction observation. Although the tensile twinning becomes active by the end of unloading in both samples, as seen in Fig. 9, the amount of grains involved in detwinning during unloading must be very few, since 
no simultaneous diffraction peak intensity variation can be detected from the neutron diffraction measurement.

In our previous paper (Wu et al., 2013), it states that no apparent detwinning process can be identified even in the elastic region during reverse tension for both samples. Although the diffraction peak intensity of (00.2) twin grains decreased in the elastic region during reverse tension in the pre-deformed sample, no simultaneous diffraction peak intensity variation was detected in (10.0) grains. This phenomenon is attributed to the grain rotation resulting from the microscopic stress relaxation and macroscopic rapid stress drop (Benafan et al., 2013; Wu et al., 2013), which could lead to the complex stress state in some localized region and favor the grain rotation. In the present paper, the EVPSC-TDT simulation results approve that no apparent detwinning occurs, as demonstrated in Fig. 8, that no obvious twin volume fraction varies in the early stage of the elastic region during reverse tension for both samples, and in Fig. 9 the basal <a> slip is the dominant plastic deformation mode during unloading. Besides that, it is also indicated in Fig. 9 that intergranular stresses drive plasticity in some grains during unloading, even prior to reaching zero applied stress.

The observation that there is no pronounced detwinning process during unloading was also made by others. For a rolled AZ31 sheet, Uota et al. (Uota et al., 2009) reported that twins were formed by compression straining. These twins were remained under unloading and disappeared under tension loading. Very recently, Lee et al. (Lee et al., 2014) studied the deformation behavior of a solid-solution-strengthened $\mathrm{Mg}-9 \mathrm{wt} \% \mathrm{Al}$ alloy under loading and unloading. It was suggested that for this extruded Mg alloy, the pseudoelastic behavior in the macroscopic stressstrain responses during unloading is due to plastic deformation by the operation of basal slip in the soft grain orientations. 
The pronounced detwinning process does not occur, until the applied stress passes the yield strength during reverse tension. A certain amount of lattice strains $(2,181$ and $2,145 \mu \varepsilon$ in the asreceived and pre-deformed samples, respectively) has to be accumulated in twin grains before detwinning, as marked in Figs. 6(g) and (h). It is thought to account for the little difference in the tensile yield strength during reverse tension between the as-received and pre-deformed samples. The EVPSC-TDT simulation results for both samples in Fig. 8 exhibit that (1) in the stress range III/iii the twin volume fraction decreased dramatically, (2) in the stress range IV/iv the trend of the decrease became gradually, and (3) in the stress range $\mathrm{V} / \mathrm{v}$ the twin volume fraction kept constant. These trends indicate that the massive detwinning process occurs in the stress range III/iii and the detwinning process is completed by the stress range IV/iv, which agrees with the experimental observation very well. For the as-received sample in Fig. 9(a), it reveals that (1) in the stress range III the detwinning process is the dominant deformation mode, (2) in the stress range IV the basal $<a>$ slip rules over other deformation modes and prismatic $<a>$ slip turns out to be more and more active while the detwinning process becomes less and less operative, and (3) in the stress range $V$ the basal $<a>$ slip and prismatic $<a>$ slip prevail and detwinning remains inactive. The pre-deformed sample has a comparable trend as the as-received sample, but there are some differences, such as in the pre-deformed sample, the detwinning deformation mode is dominant at the beginning of the stress range iv, the prismatic <a> slip is the dominant deformation mode in the middle of the stress range iv and becomes inactive in the stress range $\mathrm{v}$. 


\section{Conclusions}

The real-time in-situ neutron diffraction technique under a continuous loading condition and numerical simulations using the EVPSC-TDT model were employed to study the plasticdeformation dynamics on the twinning and detwinning behavior of the wrought $\mathrm{AZ31B} \mathrm{Mg}$ alloy. An effective relationship has been established between the macroscopic mechanical properties and the microscopic response. The following conclusions can be drawn, based on the present research:

(1) The plastic deformation can be divided into several stages during compression and reverse tension, based on the work-hardening-rate variations. In each stage, a certain deformation mode becomes dominant, which is characterized by real-time in-situ neutron diffraction and supported by simulation results.

(2) The EVPSC-TDT model was employed for the first time to predict the deformation dynamics under a continuous-loading condition during strain-path changes in a HCPstructured $\mathrm{Mg}$ alloy. The simulation results concur with the experimental observation of the alternate deformation mechanisms during strain-path changes.

(3) An obvious strengthening behavior was obtained during compression in the pre-deformed sample. However, the pre-deformation has limited impacts on mechanical behavior during reverse tension.

(4) It is evident that the residual strain is responsible for the delay of the onset of tensile twinning in the subsequent compression in the pre-deformed sample. However, by increasing the dislocation density through pre-tension, the twinning and detwinning process can be accelerated, once the twinning or detwinning process is activated. 
(5) As previously reported (Wu et al., 2013), no apparent detwinning activities were observed during unloading after compression. Detwinning does not occur until the applied stress is beyond the tensile yield strength during reverse tension.

(6) In the pre-deformed sample, grain rotation is thought to account for the diffraction peak intensity decrease in (00.2) twin grains in the elastic deformation region during reverse tension. 


\section{Acknowledgement}

The research conducted at SNS, ORNL was sponsored by the Scientific User Facilities Division, Office of Basic Energy Sciences, Department of Energy (DOE). The authors appreciate beamline scientists and staff at the VULCAN Engineering Diffractometer, SNS, ORNLfor their kind help. WW is grateful for the financial support from Columbus McKinnon Corporation and a Laboratory Directed Research and Development (LDRD) project of ORNL. HQ and PDW thank the support provided by the Natural Sciences and Engineering Research Council of Canada (NSERC). XQG was supported by the China Scholarship Council (No. 201206420031) and the State Key Development Program for Basic Research of China (Grant No. 2013CB227900). PKL very much appreciates the financial support from the US National Science Foundation (DMR0909037, CMMI-0900271, and CMMI-1100080) with C. Huber, C. V. Cooper, D. Finotello, A. Ardell, and E. Taleff as contract monitors, and DOE, Office of Fossil Energy, National Energy Technology Laboratory (DE-FE-0008855 and DE-FE-001194), with Mr. V. Cedro and S. Markovich as program managers. 


\section{References}

Abdolvand, H., Daymond, M.R., 2012. Internal strain and texture development during twinning: Comparing neutron diffraction measurements with crystal plasticity finite-element approaches. Acta Mater. 60, 2240-2248.

Aghion, E., Bronfin, B., 2000. Magnesium alloys development towards the 21(st) century, in: Kojima, Y., Aizawa, T., Kamado, S. (Eds.), Magnesium Alloys 2000. Trans Tech Publications Ltd, Zurich-Uetikon, pp. 19-28.

Agnew, S., 2004. Wrought magnesium: A 21st century outlook. Jom, 20-21.

Agnew, S., Brown, D., Vogel, S., Holden, T., 2002. In-situ measurement of internal strain evolution during deformation dominated by mechanical twinning. Ecrs 6: Proceedings of the 6th European Conference on Residual Stresses, 747-752.

Agnew, S., Nie, J., 2010. Preface to the viewpoint set on: The current state of magnesium alloy science and technology. Scripta Mater. 63, 671-673.

Agnew, S., Yoo, M., Tome, C., 2001. Application of texture simulation to understanding mechanical behavior of $\mathrm{Mg}$ and solid solution alloys containing Li or Y. Acta Mater. 49, 4277-4289.

Agnew, S.R., Duygulu, O., 2005. Plastic anisotropy and the role of non-basal slip in magnesium alloy AZ31B. Int. J. Plast. 21, 1161-1193.

Agnew, S.R., Tome, C.N., Brown, D.W., Holden, T.M., Vogel, S.C., 2003. Study of slip mechanisms in a magnesium alloy by neutron diffraction and modeling. Scr. Mater. 48, 1003-1008.

An, K., 2012. VDRIVE-data reduction and interactive visualization software for event mode neutron diffraction. ORNL Report No. ORNL-TM-2012-621 Oak Ridge National Laboratory, Oak Ridge, TN.

An, K., Skorpenske, H.D., Stoica, A.D., Ma, D., Wang, X.L., Cakmak, E., 2011. First in situ lattice strains measurements under load at VULCAN. Metall. Mater. Trans. A 42, 95-99.

Balogh, L., Brown, D.W., Mosbrucker, P., Long, F., Daymond, M.R., 2012. Dislocation structure evolution induced by irradiation and plastic deformation in the $\mathrm{Zr}-2.5 \mathrm{Nb}$ nuclear structural material determined by neutron diffraction line profile analysis. Acta Mater. 60, 5567-5577.

Barnett, M.R., 2007. Twinning and the ductility of magnesium alloys Part I: "Tension" twins. Mater. Sci. Eng. A 464, 1-7.

Benafan, O., Noebe, R.D., Padula Ii, S.A., Gaydosh, D.J., Lerch, B.A., Garg, A., Bigelow, G.S., An, K., Vaidyanathan, R., 2013. Temperature-dependent behavior of a polycrystalline NiTi shape memory alloy around the transformation regime. Scripta Mater. 68, 571-574.

Brown, D., Agnew, S., Abeln, S., Blumenthal, W., Bourke, M., Mataya, M., Tome, C., Vogel, S., 2005a. The role of texture, temperature, and strain rate in the activity of deformation twinning. Mater. Sci. Forum 495-497, 1037-1042.

Brown, D.W., Agnew, S.R., Bourke, M.A.M., Holden, T.M., Vogel, S.C., Tome, C.N., $2005 b$. Internal strain and texture evolution during deformation twinning in magnesium. Mater. Sci. Eng. A 399, 1-12.

Brown, D.W., Almer, J.D., Clausen, B., Mosbrucker, P.L., Sisneros, T.A., Vogel, S.C., $2013 a$. Twinning and de-twinning in beryllium during strain path changes. Mater. Sci. Eng. A 559, 29-39. 
Brown, D.W., Clausen, B., Sisneros, T.A., Balogh, L., Beyerlein, I.J., 2013b. In situ neutron diffraction measurements during annealing of deformed beryllium with differing initial textures. Metall. Mater. Trans. A 44, 5665-5675.

Caceres, C.H., Sumitomo, T., Veidt, M., 2003. Pseudoelastic behaviour of cast magnesium AZ91 alloy under cyclic loading-unloading. Acta Mater. 51, 6211-6218.

Chen, L., Wang, C., Wu, W., Liu, Z., Stoica, G., Wu, L., Liaw, P., 2007. Low-cycle fatigue behavior of an as-extruded AM50 magnesium alloy. Metall Mater Trans A 38, 2235-2241.

Christian, J.W., Mahajan, S., 1995. Deformation twinning. Prog. Mater. Sci. 39, 1-157.

Clausen, B., Tome, C.N., Brown, D.W., Agnew, S.R., 2008. Reorientation and stress relaxation due to twinning: Modeling and experimental characterization for Mg. Acta Mater. 56, 24562468.

Davies, C.H.J., Yi, S., Bohlen, J., Kainer, K.U., Brokmeier, H.G., 2005. Synchrotron radiation investigation of twinning in extruded magnesium alloy AZ31. Mater. Sci. Forum 495-497, 1633-1638.

Doege, E., Droder, K., 2001. Sheet metal forming of magnesium wrought alloys - formability and process technology. J. Mater. Process. Tech. 115, 14-19.

El Kadiri, H., Baird, J.C., Kapil, J., Oppedal, A.L., Cherkaoui, M., Vogel, S.C., 2013. Flow asymmetry and nucleation stresses of $\{10(1)$ over-bar2 $\}$ twinning and non-basal slip in magnesium. Int. J. Plast. 44, 111-120.

Gharghouri, M.A., Weatherly, G.C., Embury, J.D., Root, J., 1999. Study of the mechanical properties of Mg-7.7at.\% Al by in-situ neutron diffraction. Philos. Mag. A 79, 1671-1695.

Guo, X.Q., Wu, W., Wu, P.D., Qiao, H., An, K., Liaw, P.K., 2013. On the Swift effect and twinning in a rolled magnesium alloy under free-end torsion. Scr. Mater. 69, 319-322.

Hama, T., Kitamura, N., Takuda, H., 2013. Effect of twinning and detwinning on inelastic behavior during unloading in a magnesium alloy sheet. Mater. Sci. Eng. A. 583, 232-241.

Hao, H., Maijer, D., Rogge, R., 2009. Investigation of residual strains by neutron diffraction in an AZ31 direct chill cast billet. Ndt\&E Int 42, 704-712.

Herrera-Solaz, V., LLorca, J., Dogan, E., Karaman, I., Segurado, J., 2014. An inverse optimization strategy to determine single crystal mechanical behavior from polycrystal tests: Application to AZ31 Mg alloy. Int. J. Plast. 57, 1-15.

Ishihara, S., Nan, Z., Goshirna, T., 2007. Effect of microstructure on fatigue behavior of AZ31 magnesium alloy. Mater. Sci. Eng. A 468, 214-222.

Jain, A., Duygulu, O., Brown, D., Tome, C., Agnew, S., 2008. Grain size effects on the tensile properties and deformation mechanisms of a magnesium alloy, AZ31B, sheet. Mater. Sci. Eng. A 486, 545-555.

Jiang, L., Jonas, J.J., Mishra, R.K., Luo, A.A., Sachdev, A.K., Godet, S., 2007. Twinning and texture development in two $\mathrm{Mg}$ alloys subjected to loading along three different strain paths. Acta Mater. 55, 3899-3910.

Khan, A.S., Pandey, A., Gnaupel-Herold, T., Mishra, R.K., 2011. Mechanical response and texture evolution of AZ31 alloy at large strains for different strain rates and temperatures. Int. J. Plast. 27, 688-706.

Khosravani, A., Scott, J., Miles, M.P., Fullwood, D., Adams, B.L., Mishra, R.K., 2013. Twinning in magnesium alloy AZ31B under different strain paths at moderately elevated temperatures. Int. J. Plast. 45, 160-173.

Kim, H.K., Lee, Y.I., Chung, C.S., 2005. Fatigue properties of a fine-grained magnesium alloy produced by equal channel angular pressing. Scripta Mater. 52, 473-477. 
Knezevic, M., Beyerlein, I.J., Brown, D.W., Sisneros, T.A., Tome, C.N., 2013. A polycrystal plasticity model for predicting mechanical response and texture evolution during strain-path changes: Application to beryllium. Int. J. Plast. 49, 185-198.

Lebensohn, R.A., Tome, C.N., 1993. A self-consistent anisotropic approach for the simulation of plastic-deformation and texture development of polycrystals - application to zirconium alloys. Acta Metall. Mater. 41, 2611-2624.

Lee, S.Y., Wang, H., Gharghouri, M.A., Nayyeri, G., Woo, W., Shin, E., Wu, P.D., Poole, W.J., Wu, W., An, K., 2014. Deformation behavior of solid-solution-strengthened Mg-9 wt.\% Al alloy: In situ neutron diffraction and elastic-viscoplastic self-consistent modeling. Acta Mater. 73, 139-148.

Liu, W.C., Wu, G.H., Zhai, C.Q., Ding, W.J., Korsunsky, A.M., 2013. Grain refinement and fatigue strengthening mechanisms in as-extruded $\mathrm{Mg}-6 \mathrm{Zn}-0.5 \mathrm{Zr}$ and $\mathrm{Mg}-10 \mathrm{Gd}-3 \mathrm{Y}-0.5 \mathrm{Zr}$ magnesium alloys by shot peening. Int. J. Plast. 49, 16-35.

Liu, Y., Wei, Y.J., 2014. A polycrystal based numerical investigation on the temperature dependence of slip resistance and texture evolution in magnesium alloy AZ31B. Int. J. Plast. $55,80-93$.

Lou, X.Y., Li, M., Boger, R.K., Agnew, S.R., Wagoner, R.H., 2007. Hardening evolution of AZ31B Mg sheet. Int. J. Plast. 23, 44-86.

Ma, Q., El Kadiri, H., Oppedal, A.L., Baird, J.C., Li, B., Horstemeyer, M.F., Vogel, S.C., 2012. Twinning effects in a rod-textured AM30 Magnesium alloy. Int. J. Plast. 29, 60-76.

Molinari, A., Canova, G.R., Ahzi, S., 1987. A Self-Consistent Approach of the Large Deformation Polycrystal Viscoplasticity. Acta Metall. Mater. 35, 2983-2994.

Mordike, B., Ebert, T., 2001. Magnesium - Properties - Applications - Potential. Mater. Sci. Eng. A $302,37-45$.

Muransky, O., Barnett, M.R., Carr, D.G., Vogel, S.C., Oliver, E.C., 2010. Investigation of deformation twinning in a fine-grained and coarse-grained ZM20 Mg alloy: Combined in situ neutron diffraction and acoustic emission. Acta Mater. 58, 1503-1517.

Muransky, O., Carr, D.G., Sittner, P., Oliver, E.C., 2009. In situ neutron diffraction investigation of deformation twinning and pseudoelastic-like behaviour of extruded AZ31 magnesium alloy. Int. J. Plast. 25, 1107-1127.

Muransky, O., Daymond, M.R., Bhattacharyya, D., Zanellato, O., Vogel, S.C., Edwards, L., 2013. Load partitioning and evidence of deformation twinning in dual-phase fine-grained $\mathrm{Zr}$ $2.5 \% \mathrm{Nb}$ alloy. Mater. Sci. Eng. A 564, 548-558.

Muranskya, O., Carr, D.G., Barnett, M.R., Oliver, E.C., Sittner, P., 2008. Investigation of deformation mechanisms involved in the plasticity of AZ31 Mg alloy: In situ neutron diffraction and EPSC modelling. Mater. Sci. Eng. A 496, 14-24.

Park, S.C., Lim, J.D., Eliezer, D., Shin, K.W., 2003. Microstructure and mechanical properties of Mg-Zn-Ag alloys, in: Kojima, Y., Aizawa, T., Higashi, K., Kamado, S. (Eds.), Magnesium Alloys 2003, Pts 1 and 2. Trans Tech Publications Ltd, Zurich-Uetikon, pp. 159-164.

Polmear, I.J., 1994. Magnesium alloys and applications. Mater. Sci. Technol. 10, 1-16.

Prakash, D.G.L., Honniball, P., Rugg, D., Withers, P.J., da Fonseca, J.Q., Preuss, M., 2013. The effect of beta phase on microstructure and texture evolution during thermomechanical processing of alpha plus beta Ti alloy. Acta Mater. 61, 3200-3213.

Proust, G., Tomé, C.N., Jain, A., Agnew, S.R., 2009. Modeling the effect of twinning and detwinning during strain-path changes of magnesium alloy AZ31. Int. J. Plast. 25, 861-880.

Roberts, C.S., 1960. Magnesium and its alloys. John Wiley \& Sons, Inc, New York. 
Skippon, T., Clausen, B., Daymond, M.R., 2013. Effect of loading mode on lattice strain measurements via neutron diffraction. Mater. Sci. Eng. A 577, 169-178.

Stanford, N., Barnett, M.R., 2013. Solute strengthening of prismatic slip, basal slip and \{1 0 (1)over-bar 2$\}$ twinning in Mg and Mg-Zn binary alloys. Int. J. Plast. 47, 165-181.

Turner, P.A., Tome, C.N., 1994. A study of residual-stresses in Zircaloy-2 with rod texture. Acta Metall. Mater. 42, 4143-4153.

Uota, T., Suzu, T., Fukumoto, S., Yamamoto, A., 2009. EBSD observation for reversible behavior of deformation twins in AZ31B magnesium alloy. Mater. Trans. A 50, 2118-2120.

Wang, H., Raeisinia, B., Wu, P.D., Agnew, S.R., Tome, C.N., 2010a. Evaluation of selfconsistent polycrystal plasticity models for magnesium alloy AZ31B sheet. Int. J. Solids Struct. 47, 2905-2917.

Wang, H., Wu, P.D., Boyle, K.P., Neale, K.W., 2011. On crystal plasticity formability analysis for magnesium alloy sheets. Inter. J. Solids Struct. 48, 1000-1010.

Wang, H., Wu, P.D., Gharghouri, M.A., 2010b. Effects of basal texture on mechanical behaviour of magnesium alloy AZ31B sheet. Mater. Sci. Eng. A 527, 3588-3594.

Wang, H., Wu, P.D., Tomé, C.N., Huang, Y., 2010c. A finite strain elastic-viscoplastic selfconsistent model for polycrystalline materials. J. Mech. Phys. Solids 58, 594-612.

Wang, H., Wu, P.D., Tomé, C.N., Wang, J., 2012a. A constitutive model of twinning and detwinning for hexagonal close packed polycrystals. Mater. Sci. Eng. A 555, 93-98.

Wang, H., Wu, P.D., Tomé, C.N., Wang, J., 2012b. Study of lattice strains in magnesium alloy AZ31 based on a large strain elastic-viscoplastic self-consistent polycrystal model. Int. J. Solids Struct. 49, 2155-2167.

Wang, H., Wu, P.D., Wang, J., 2013a. Modeling inelastic behavior of magnesium alloys during cyclic loading-unloading. Int. J. Plast. 47, 49-64.

Wang, H., Wu, P.D., Wang, J., Tome, C.N., 2013b. A crystal plasticity model for hexagonal close packed (HCP) crystals including twinning and de-twinning mechanisms. Int. J. Plast. 49, 36-52.

Wang, J., Beyerlein, I.J., Tome, C.N., 2014. Reactions of lattice dislocations with grain boundaries in $\mathrm{Mg}$ : Implications on the micro scale from atomic-scale calculations. Int $\mathrm{J}$ Plasticity 56, 156-172.

Wang, X.L., Holden, T.M., Rennich, G.Q., Stoica, A.D., Liaw, P.K., Choo, H., Hubbard, C.R., 2006. VULCAN - The engineering diffractometer at the SNS. Physica B 385, 673-675.

Wang, X.L., Holden, T.M., Stoica, A.D., AN, K., Skorpenske, H.D., Jones, A.B., Rennich, G.Q., Iverson, E.B., 2010d. First results from the VULCAN diffractometer at the SNS. Mater. Sci. Forum 652, 105-110.

Wang, X.L., Stoica, A.D., 2009. Focusing neutron guides for VULCAN-Design aspects, estimated performance, and detector deployment. Nucl. Instrum. Meth. A 600, 309-312.

Withers, P.J., Clarke, A.P., 1998. A neutron diffraction study of load partitioning in continuous $\mathrm{Ti} / \mathrm{SiC}$ composites. Acta Mater. 46, 6585-6598.

Woo, W., Choo, H., Prime, M.B., Feng, Z., Clausen, B., 2008. Microstructure, texture and residual stress in a friction-stir-processed AZ31B magnesium alloy. Acta Mater. 56, 17011711.

Wu, B.L., Wan, G., Zhang, Y.D., Esling, C., 2010. Twinning characteristics in textured AZ31 alloy under impact loading along specified direction. Mater. Lett. 64, 636-639. 
Wu, L., 2009. Mechanical behavior and the role of deformation twinning in wrought magnesium alloys investigated using neutron and synchrotron X-ray diffraction, In: Materials Science and Engineering. The University of Tennessee, Knoxville.

Wu, L., Agnew, S.R., Brown, D.W., Stoica, G.M., Clausen, B., Jain, A., Fielden, D.E., Liaw, P.K., 2008a. Internal stress relaxation and load redistribution during the twinningdetwinning-dominated cyclic deformation of a wrought magnesium alloy, ZK60A. Acta Mater. 56, 3699-3707.

Wu, L., Jain, A., Brown, D.W., Stoica, G.M., Agnew, S.R., Clausen, B., Fielden, D.E., Liaw, P.K., 2008b. Twinning-detwinning behavior during the strain-controlled low-cycle fatigue testing of a wrought magnesium alloy, ZK60A. Acta Mater. 56, 688-695.

Wu, W., An, K., Huang, L., Lee, S.Y., Liaw, P.K., 2013. Deformation dynamics study of a wrought magnesium alloy by real-time in situ neutron diffraction. Scripta Mater. 69, 358361.

Wu, W., Lee, S.Y., Paradowska, A.M., Gao, Y., Liaw, P.K., 2012. Twinning-detwinning behavior during fatigue-crack propagation in a wrought magnesium alloy AZ31B. Mater. Sci. Eng. A 556, 278-286.

Yang, F., Yin, S.M., Li, S.X., Zhang, Z.F., 2008. Crack initiation mechanism of extruded AZ31 magnesium alloy in the very high cycle fatigue regime. Mater. Sci. Eng. A 491, 131-136.

Yu, Q., Qi, L., Chen, K., Mishra, R.K., Li, J., Minor, A.M., 2012. The nanostructured origin of deformation twinning. Nano Lett. 12, 887-892. 


\section{Table Titles}

Table 1. List of material parameters for slip and twin systems used in the EVPSC-TDT model. The parameter, $h^{s t}$, lists latent hardening of the twinning activity upon other slip modes. All other latent-hardening parameters are 1.

\section{Figure Captions}

Figure 1. Schematic of a real-time in-situ neutron diffraction setup at VULCAN, SNS, ORNL (Wu et al., 2013).

Figure 2. The two-dimensional (2-D) real-time in-situ neutron diffraction results of the measured d range during strain-path changes for: (a) the as-received sample in the axial direction, (b) the as-received sample in the radial direction, (c) the pre-deformed sample in the axial direction, and (d) the pre-deformed sample in the radial direction. The color bars represent the maximum height of diffraction peaks.

Figure 3. Monotonic uniaxial tension and compression of the as-received material along the RD. (a) Measured (open symbols) and fitted stress-strain curves (dashed lines); (b) Relative activities of various slip and twinning mechanisms.

Figure 4. The stress vs. strain curves during strain-path changes for: (a) the as-received sample and (b) the pre-deformed sample (Wu et al., 2013). The loading sequence was marked by the orange arrows and circled numbers. The inflection points of the stress-strain curves were marked by colored dots.

Figure 5. The diffraction peak intensity evolution of certain $h k l s$ as a function of stress in the axial direction from real-time in-situ neutron diffraction measurements (open symbols) and simulations (dashed lines) for: (a) the as-received sample and (b) the pre-deformed sample.

Figure 6. The internal-strain evolutions of certain $h \mathrm{kl}$ s from real-time in-situ neutron diffraction measurements. (a), (c), (e), and (g) represent the lattice-strain evolution of the asreceived sample, and (b), (d), (f), and (h) are the lattice-strain evolution of the predeformed sample during strain-path changes.

Figure 7. Measured (open symbols) and calculated (dashed lines) lattice strains in the axial direction as a function of applied stress for both the as-received (left) and pre-strained (right) samples

Figure 8. The simulation results of the twin-volume-fraction variation, $f^{t w}$, during strain-path changes for: (a) the as-received sample and (b) the pre-deformed sample. 
Figure 9. The relative activities of interested deformation modes during strain-path changes from the EVPSC-TDT model for: (a) the as-received sample and (b) the pre-deformed sample. 